\title{
A biomimetic domino reaction for the concise synthesis of capreomycidine and epicapreomycidine
}

\author{
Martin Büschleb • Markus Granitzka • \\ Dietmar Stalke $\cdot$ Christian Ducho
}

Received: 31 March 2012/Accepted: 19 April 2012/Published online: 23 May 2012

(C) Springer-Verlag 2012

\begin{abstract}
The non-proteinogenic amino acids capreomycidine and epicapreomycidine are constituents of antibiotically active natural products, but the synthesis of these unusual cyclic guanidine derivatives is challenging. The biosynthesis of capreomycidine has therefore been employed as a guideline to develop a concise biomimetic synthesis of both epimeric amino acids. The resulting domino-guanidinylation-azaMichael-addition reaction provides the most convenient access to these amino acids in racemic form. Attempts to dissect the domino reaction into two separate transformations for a stereocontrolled version of this synthetic approach have also been made. The synthesized didehydro-arginine derivatives with urethane-protected guanidine moieties did not undergo the aza-Michael-addition anymore. These results may have wider implications for the 1,4-addition of guanidines to $\alpha, \beta$-unsaturated carbonyl compounds, particularly to didehydro amino acids.
\end{abstract}

Electronic supplementary material The online version of this article (doi:10.1007/s00726-012-1309-8) contains supplementary material, which is available to authorized users.

\section{Büschleb · C. Ducho}

Department of Chemistry, Institute of Organic and Biomolecular Chemistry, Georg-August-University Göttingen, Tammannstr. 2, 37077 Göttingen, Germany

\section{Granitzka · D. Stalke}

Department of Chemistry, Institute of Inorganic Chemistry, Georg-August-University Göttingen, Tammannstr. 4,

37077 Göttingen, Germany

\section{Ducho $(\square)$}

Department of Chemistry, University of Paderborn,

Warburger Str. 100, 33098 Paderborn, Germany

e-mail: christian.ducho@uni-paderborn.de

URL: http://chemie.uni-paderborn.de/fachgebiete/oc/ak-ducho
Keywords Natural products - Antibiotics - Amino acids · Biomimetic synthesis · Domino reactions · Guanidines

\section{Introduction}

Bacterial infections continue to be a major threat to human health, particularly with respect to emerging strains with resistances to established antibiotics (Taubes 2008; Cooper and Shlaes 2011). It is therefore highly desirable to develop novel antibacterial agents which should ideally display new or yet unexploited modes of action (Walsh 2003). Natural products have been used as lead structures for many antibacterial drugs (Mahady et al. 2008). However, their often complex structures represent important challenges to organic synthesis as a prerequisite for detailed structureactivity relationship (SAR) studies. Among the many bacterial secondary metabolites with antibiotic activity, a significant number of compounds are of peptidic nature and contain non-proteinogenic amino acid structures.

The cyclic arginine-derived non-proteinogenic amino acids $(2 S, 3 R)$-capreomycidine $\mathbf{1}$ ('capreomycidine') and $(2 S, 3 S)$-capreomycidine $\mathbf{2}$ ('epicapreomycidine') are constituents of several natural products with remarkable antibiotic potencies (Fig. 1). Capreomycidine $\mathbf{1}$ can be found in tuberactinomycin peptide antibiotics (Nagata et al. 1968; Wakamiya et al. 1970; Yoshioka et al. 1971; Ando et al. 1971; Izumi et al. 1972), e.g., the capreomycins (Herr et al. 1960; Bycroft et al. 1971a; Shiba et al. 1976; Nomoto et al. 1977; Nomoto et al. 1978) and viomycin 3 (Finlay et al. 1951; Bartz et al. 1951; Dyer et al. 1965; Bycroft et al. 1969; Noda et al. 1972), which have both found clinical use as anti-tuberculosis drugs. The 3-epimer epicapreomycidine $\mathbf{2}$ is a component of the naturally occurring protease inhibitors chymostatin (Umezawa et al. 1970; Tatsuta et al. 
1973) and elastatinal (Umezawa et al. 1973; Okura et al. 1975) as well as of the Streptomyces-produced muraymycins, a subclass of nucleoside lipopeptide antibiotics (e.g. muraymycin A1 4, McDonald et al. 2002). Furthermore, $\beta$-hydroxy-enduracididine, an amino acid unit with structural similarities to $\mathbf{1}$ and $\mathbf{2}$, is a building block of the mannopeptimycins, a novel subclass of glycopeptide antibiotics (He et al. 2002; Singh et al. 2003; Koehn 2008; Schwörer and Oberthür 2009).

Due to their relevance in natural product chemistry, several approaches for the synthesis of amino acids $\mathbf{1}$ and $\mathbf{2}$ have been developed. A first non-stereoselective synthesis of $\mathbf{1}$ and $\mathbf{2}$ involved the preparation of an aromatic 2-aminopyrimidine precursor, which was then hydrogenated to provide a diastereomeric mixture of $\mathbf{1}$ and $\mathbf{2}$, each in racemic form. The separation of the diastereomers was carried out by crystallization of the respective picrates (Bycroft et al. 1971b). A slightly improved version of this synthesis has also been described (Yamashita et al. 2004). The first syntheses of stereochemically pure $\mathbf{1}$ and $\mathbf{2}$ employed an aldol reaction for the synthesis of a protected $\beta$-hydroxy-ornithine precursor, which then underwent acylase-mediated enzymatic resolution. Further conversion via an aziridine intermediate finally provided either $\mathbf{1}$ or $\mathbf{2}$, but the synthesis was lengthy, and the overall yields were rather low (Shiba et al. 1977; Wakamiya et al. 1978; Teshima et al. 1980). The first stereoselective synthesis of capreomycidine $\mathbf{1}$ involved a Mannich-type reaction with a chiral glycine derivative as the key step. The subsequent guanidinylation reaction only proceeded with one of the two diastereomers obtained from the Mannich transformation, thus finally giving pure $\mathbf{1}$ after further conversions (DeMong and Williams 2001, 2003). However, this also implies that an according synthesis of $\mathbf{2}$ using the same strategy is not feasible. An alternative ex-chiral pool synthesis of ${ }^{13} \mathrm{C}$-labelled 1 starting from Garner's aldehyde has also been established (Jackson et al. 2002), but investigations in our own laboratory revealed that an application of this approach to the synthesis of $\mathbf{2}$ is not possible (unpublished data). The first practical synthesis of stereochemically pure epicapreomycidine has been established in recent years. The initial version of this synthetic route involved an ornithine-derived sulfamate which was then employed in a rhodium-catalyzed $\mathrm{C}-\mathrm{H}$ insertion reaction to construct the stereocenter in the C-3 position. However, this elegant approach suffered from low yields and diastereoselectivities of the $\mathrm{C}-\mathrm{H}$ activation key step (Tanino et al. 2008, 2010). A superior version of this $\mathrm{C}-\mathrm{H}$ activation strategy starting from D-tyrosine and displaying perfect stereocontrol has recently been described though (Tanino et al. 2011).

One of the highly useful strategies for the development of efficient synthetic routes is to imitate the biosynthetic pathways found in nature. Such biomimetic syntheses have found widespread applications (Razzak and De Brabander 2011). Our goal was to investigate the feasibility of a biomimetic synthesis of both capreomycidines $\mathbf{1}$ and $\mathbf{2}$. The biosynthetic assembly of the cyclic guanidine structure of capreomycidine $\mathbf{1}$ as part of viomycin biosynthesis in Streptomyces vinaceus has been elucidated (Fig. 2, Yin and Zabriskie 2004; Yin et al. 2004; Ju et al. 2004). Stereoselective 3-hydroxylation of L-arginine $\mathbf{5}$, catalyzed by the non-heme 2-oxoglutarate (2-OG) dependent $\mathrm{Fe}(\mathrm{II})$-oxygenase VioC, provides (3S)-3-hydroxy-L-arginine 6. The subsequent ring closure reaction is mediated by the pyridoxalphosphate-(PLP)-dependent enzyme VioD and most likely proceeds via the $\alpha, \beta$-unsaturated didehydro-arginine intermediate 7, as demonstrated by labelling studies. Michael-type conjugate 1,4-addition of the guanidine moiety then stereoselectively provides capreomycidine $\mathbf{1}$<smiles>N=C1NCC[C@H]([C@H](N)C(=O)O)N1</smiles><smiles>N=C1C[C@@H](N)CCN1</smiles>

2

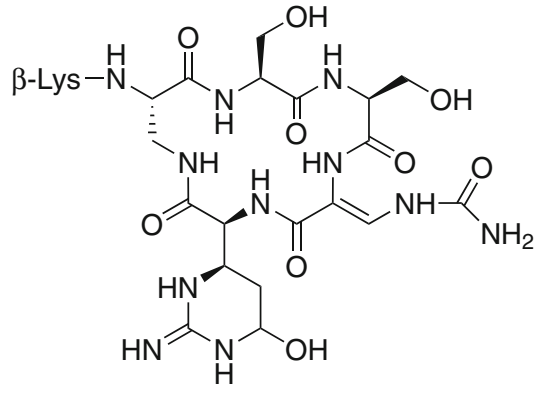

3<smiles>[Y19]C(C)C(=O)C[Y1]([H])([H])CN(O)C(=N)N</smiles>

Fig. 1 Structures of non-proteinogenic amino acids capreomycidine $\mathbf{1}$ and epicapreomycidine $\mathbf{2}$ as well as structures of natural products viomycin 3 and muraymycin A1 $\mathbf{4}$ containing $\mathbf{1}$ and 2, respectively 
with overall formal inversion of the stereocenter at C-3. The biosynthesis of $\mathbf{2}$ has not been elucidated yet, but the recent analysis of the biosynthetic gene cluster of muraymycin nucleoside antibiotics suggests that it probably occurs in a similar manner, just with a different stereochemical course (Cheng et al. 2011; also see Lemke et al. 2010).

Hence, the goal of this study was to synthesize didehydro-arginine derivatives $\mathbf{8 a}-\mathbf{h}$ as potential precursors for biomimetic Aza-Michael-additions in order to obtain amino acids $\mathbf{1}$ and $\mathbf{2}$ or protected derivatives thereof (Table 1). There is very limited precendent for 1,4 -additions of protected guanidines, with a striking example provided by Baran, Seiple and coworkers as part of their synthetic studies on palau'amine and related compounds<smiles>N=C(N)NCCC[C@H](N)C(=O)O</smiles>

5

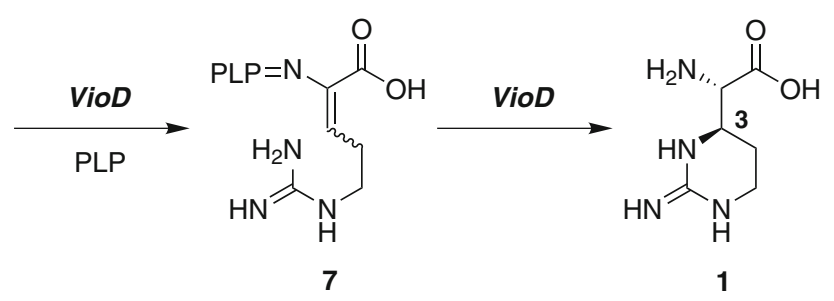

Fig. 2 Biosynthesis of capreomycidine 1 in Streoptomyces vinaceus
(Seiple et al. 2011). It was thus envisaged to investigate different protecting group patterns both at the guanidine group (to influence its nucleophilicity) and the didehydro amino acid moiety (to tune its electrophilicity).

\section{Materials and methods}

Chemicals were purchased from Sigma-Aldrich, Alfa Aesar, ABCR and VWR. Amino acid phosphonates 9a-c and 3-azido-propionaldehyde $\mathbf{1 0}$ were synthesized as previously reported (Ducho et al. 2009; Schmidt et al. 1984; Davies et al. 1967). Reactions involving oxygen and/or moisture-sensitive reagents were carried out under an atmosphere of argon using anhydrous solvents. Anhydrous solvents were obtained in the following manner: THF was dried over sodium/benzophenone and distilled, $\mathrm{MeCN}$ and $\mathrm{CH}_{2} \mathrm{Cl}_{2}$ were dried over $\mathrm{P}_{2} \mathrm{O}_{5}$ and distilled and DMF was dried over molecular sieves $4 \AA$. All other solvents were of technical quality and distilled prior to their use, and distilled water was used throughout. Column chromatography was carried out on silica gel $60 \quad(0.040-0.063 \mathrm{~mm}$, 230-400 mesh ASTM, VWR) except where indicated under flash conditions. TLC was performed on aluminium plates precoated with silica gel $60 F_{254}$ (VWR). Visualisation of the spots was carried out using UV light $(254 \mathrm{~nm})$ where appropriate and/or $\mathrm{KMnO}_{4}$ staining under heating (staining solution: $1 \mathrm{~g} \mathrm{KMnO}_{4}, 6 \mathrm{~g} \mathrm{~K}_{2} \mathrm{CO}_{3}$ and $1.5 \mathrm{~mL} 5 \% \mathrm{NaOH}_{(\mathrm{aq})}(\mathrm{w} / \mathrm{v})$, all dissolved in $100 \mathrm{~mL}$ $\mathrm{H}_{2} \mathrm{O}$ ). $300 \mathrm{MHz}^{1} \mathrm{H}$ as well as $75 \mathrm{MHz}-, 76 \mathrm{MHz}-$ and $126 \mathrm{MHz}-{ }^{13} \mathrm{C}$ NMR spectra were recorded on Varian UNITY 300, MERCURY 300, INOVA 500 and INOVA

Table 1 Target structures $\mathbf{8 a}-\mathbf{h}$ envisaged as precursors for biomimetic cyclization reactions in this study<smiles>[R7]N=C(N[R])N([R7])CCC=C(N[R7])C(=O)O[2H]</smiles>

8a-h

\begin{tabular}{lllllll}
\hline Compd. & $\mathrm{R}^{1}$ & $\mathrm{R}^{2}$ & $\mathrm{R}^{3}$ & $\mathrm{R}^{4}$ & $\mathrm{R}^{5}$ & \\
\hline $\mathbf{8 a}$ & $\mathrm{Boc}$ & $\mathrm{H}$ & $t$-Bu & $\mathrm{H}$ & $\mathrm{H}$ & $\mathrm{H}$ \\
$\mathbf{8 b}$ & $\mathrm{Boc}$ & $\mathrm{Boc}$ & $t$-Bu & $\mathrm{H}$ & $\mathrm{H}$ & $\mathrm{H}$ \\
$\mathbf{8 c}$ & $\mathrm{Ac}$ & $\mathrm{H}$ & $t$-Bu & $\mathrm{H}$ & $\mathrm{H}$ & $\mathrm{H}$ \\
$\mathbf{8 d}$ & $\mathrm{Ac}$ & $\mathrm{H}$ & $\mathrm{Me}$ & $\mathrm{Cbz}$ & $\mathrm{Cbz}$ & $\mathrm{H}$ \\
$\mathbf{8 e}$ & $\mathrm{Boc}$ & $\mathrm{H}$ & $\mathrm{Cbz}$ & $\mathrm{Cbz}$ \\
$\mathbf{8 f}$ & $\mathrm{Hoc}$ & $\mathrm{H}$ & $\mathrm{Cbz}$ & $\mathrm{B}$ \\
$\mathbf{8 g}$ & $\mathrm{Boc}$ & $\mathrm{Boc}$ & $t$-Bu & $\mathrm{Cbz}$ & $\mathrm{Cbz}$ \\
$\mathbf{8 h}$ & $\mathrm{Boc}$ & $t$-Bu & $\mathrm{Cbz}$ & $\mathrm{Bn}$ \\
\hline
\end{tabular}


600 spectrometers. All ${ }^{13} \mathrm{C}$ NMR spectra are ${ }^{1} \mathrm{H}$-decoupled. All spectra were recorded at room temperature except of samples in DMSO- $d_{6}$ and $\mathrm{D}_{2} \mathrm{O}$ (standard $35{ }^{\circ} \mathrm{C}$ ) and where indicated otherwise and were referenced internally to solvent reference frequencies. Chemical shifts $(\delta)$ are quoted in ppm. Coupling constants $(J)$ are reported in $\mathrm{Hz}$ to the nearest $0.1 \mathrm{~Hz}$. Assignment of signals was carried out using ${ }^{1} \mathrm{H},{ }^{1} \mathrm{H}-\mathrm{COSY}$ and HSQC spectra obtained on the spectrometers mentioned above. Low-resolution ESI mass spectrometry was performed on a Varian MAT 311 A spectrometer operating in positive ionization mode. High-resolution (HR) ESI mass spectrometry was carried out on a Bruker microTOF spectrometer or a Bruker 7 T FTICR APEX IV spectrometer. Melting points (mp) were measured on a Büchi instrument and are not corrected. Infrared spectroscopy (IR) was performed on a JASCO FT/IR-4100 spectrometer equipped with an ATR unit. Wavenumbers $(v)$ are quoted in $\mathrm{cm}^{-1}$. UV spectroscopy was carried out on a JASCO V-630 spectrometer. Wavelengths of maximum absorption $\left(\lambda_{\max }\right)$ are reported in $\mathrm{nm}$ with the corresponding logarithmic molar extinction coefficient $(\log \varepsilon)$ given in parenthesis.

General procedure A (synthesis of protected didehydro amino acids by Wittig-Horner reactions)

To a solution of $\mathrm{KO} t-\mathrm{Bu}$ in THF, a solution of the amino acid phosphonate in THF was added at $-78{ }^{\circ} \mathrm{C}$. After 15 min, a solution of 3-azido-propionaldehyde $\mathbf{1 0}$ in THF was added dropwise at $-78{ }^{\circ} \mathrm{C}$. The reaction mixture was stirred overnight and slowly warmed to rt during this period. The reaction was quenched by addition of $\mathrm{MeOH}$. After the addition of EtOAc, the organic layer was washed with water, dried over $\mathrm{Na}_{2} \mathrm{SO}_{4}$ and evaporated under reduced pressure. The resultant crude product was purified by column chromatography.

General procedure B (sequence of Staudinger reduction and guanidinylation reactions with reagent 13)

To a solution of the protected $\delta$-azido-didehydro $\alpha$-amino acid in THF and water, $\mathrm{PPh}_{3}$ was added at rt. The reaction mixture was stirred overnight at $\mathrm{rt}$. After the addition of EtOAc, the organic layer was washed with aqueous $\mathrm{AcOH}(10 \%, 3 \times)$. The combined aqueous layers were adjusted to $\mathrm{pH} 12$ by the addition of diluted $\mathrm{NaOH}$ solution and extracted with $\mathrm{CH}_{2} \mathrm{Cl}_{2}(3 \times)$ and $\mathrm{CH}_{2} \mathrm{Cl}_{2} / i$ $\mathrm{PrOH}(3: 1,1 \times)$. The combined organics were dried over $\mathrm{Na}_{2} \mathrm{SO}_{4}$ and evaporated under reduced pressure. The resultant crude products were sufficiently pure $(>95 \%$ as judged by ${ }^{1} \mathrm{H}$ NMR) without further purification. With respect to the limited stability of the didehydro- ornithines, they were prepared freshly and directly used for the subsequent guanidinylation reaction. Thus, the crude didehydro-ornithines were dissolved in DMF, and $\mathrm{NEt}_{3}$ and guanidinylation reagent $\mathbf{1 3}$ were added at rt. The reaction mixture was stirred at $\mathrm{rt}$ or elevated temperature (to drive the reaction to completion) for the indicated time. The solvent was evaporated under reduced pressure and the resultant crude product was purified by column chromatography.

General procedure $\mathrm{C}$ (guanidinylation reactions with reagent 31)

To a solution of the crude ornithine derivative in DMF, guanidinylation reagent $\mathbf{3 1}, \mathrm{NEt}_{3}$ and AgOTf were added. The reaction mixture was stirred at $\mathrm{rt}$ for $3 \mathrm{~h}$, then filtered through Celite and the Celite were washed with EtOAc $(3 \times)$. The filtrate was washed with brine $(3 \times)$, dried over $\mathrm{Na}_{2} \mathrm{SO}_{4}$ and evaporated under reduced pressure. The resultant crude product was purified by column chromatography.

Capreomycidine dihydrochloride $((\mathrm{rac})-\mathbf{1} \cdot 2 \mathrm{HCl})$ and epicapreomycidine dihydrochloride

$((\mathrm{rac})-\mathbf{2} \cdot 2 \mathrm{HCl})$

\section{From (rac)-14 and (rac)-15}

A solution of a mixture of protected capreomycidine (rac)-14 and epicapreomycidine (rac)-15 $(580 \mathrm{mg}$, $1.77 \mathrm{mmol})$ in aqueous $\mathrm{HCl}(6 \mathrm{M}, 10 \mathrm{~mL}, 60 \mathrm{mmol})$ was stirred at $\mathrm{rt}$ for $4 \mathrm{~h}$. The solvent was evaporated under reduced pressure to give a mixture of $(\mathrm{rac})-\mathbf{1} \cdot 2 \mathrm{HCl}$ and (rac)-2 $\cdot 2 \mathrm{HCl}$ as a slightly brownish oil $(430 \mathrm{mg}, 99 \%)$. ${ }^{1} \mathrm{H}$ NMR $\left(300 \mathrm{MHz}, \mathrm{D}_{2} \mathrm{O}\right): \delta=1.90-2.23(\mathrm{~m}, 2 \times 2 \mathrm{H}$, H-4), 3.36-3.55 (m, $2 \times 2 \mathrm{H}, \mathrm{H}-5), 4.18-4.30$ (m, $2 \times 2 \mathrm{H}, \mathrm{H}-2, \mathrm{H}-3) .{ }^{13} \mathrm{C}$ NMR $\left(76 \mathrm{MHz}, \mathrm{D}_{2} \mathrm{O}\right): \delta=20.7$ $(1 \times \mathrm{C}-4), 21.6(1 \times \mathrm{C}-4), 36.0(1 \times \mathrm{C}-5), 36.4(1 \times \mathrm{C}-$ 5), $48.4(1 \times \mathrm{C}-3), 48.7(1 \times \mathrm{C}-3), 55.3(1 \times \mathrm{C}-2), 55.4$ $(1 \times \mathrm{C}-2), 154.5(2 \times$ guanidine-C $), 169.2(1 \times \mathrm{C}=\mathrm{O})$, $169.5(1 \times \mathrm{C}=\mathrm{O}) . \mathrm{MS}\left(\mathrm{ESI}^{+}\right): m / z=173.1[\mathrm{M}+\mathrm{H}]^{+}$. HRMS $\left(\mathrm{ESI}^{+}\right)$: calcd. for $\mathrm{C}_{6} \mathrm{H}_{12} \mathrm{~N}_{4} \mathrm{O}_{2} \quad\left([\mathrm{M}+\mathrm{H}]^{+}\right)$ 173.1033, found 173.1034 .

\section{From (rac)-17 and (rac)-18}

A solution of protected capreomycidine $(\mathrm{rac})-\mathbf{1 7}$ and epicapreomycidine $(\mathrm{rac})-\mathbf{1 8}(75 \mathrm{mg}, 0.18 \mathrm{mmol})$ in aqueous $\mathrm{HCl}(6 \mathrm{M}, 1.8 \mathrm{~mL}, 11 \mathrm{mmol})$ was stirred at $\mathrm{rt}$ for $3 \mathrm{~h}$. The solvent was evaporated under reduced pressure to give a mixture of $(\mathrm{rac})-\mathbf{1} \cdot 2 \mathrm{HCl}$ and $(\mathrm{rac})-\mathbf{2} \cdot 2 \mathrm{HCl}$ as a slightly brownish oil (37 mg, $87 \%$ ). Analytical data were identical to those given above. 
$N^{2}$-Acetyl-2,3-didehydro-arginine tert-butyl ester (8c)

General procedure B with (Z)-tert-butyl $N^{2}$-acetyl-2-amino-5azido-pent-2-enoate $(Z)-\mathbf{1 1 b}\left(334 \mathrm{mg}, 1.32 \mathrm{mmol}\right.$ ), $\mathrm{PPh}_{3}$ $(689 \mathrm{mg}, 2.63 \mathrm{mmol})$, water $(1.3 \mathrm{~mL})$ and THF $(13 \mathrm{~mL})$ for the Staudinger reduction as well as guanidinylation reagent $\mathbf{1 3}$ (386 mg, $2.63 \mathrm{mmol}$ ), $\mathrm{NEt}_{3}(266 \mathrm{mg}, 364 \mu \mathrm{L}, 2.63 \mathrm{mmol}$ ) and DMF $(13 \mathrm{~mL})$ for the guanidinylation reaction. The reaction mixture was stirred at $\mathrm{rt}$ for 8 days and at $100{ }^{\circ} \mathrm{C}$ for $22 \mathrm{~h}$. Evaporation of the solvent under reduced pressure gave the crude product of $\mathbf{8 c}$ as yellow oil $(417 \mathrm{mg}) .{ }^{1} \mathrm{H}$ NMR $\left(300 \mathrm{MHz}, \mathrm{DMSO}-\mathrm{d}_{6}\right): \delta=1.39\left(\mathrm{~s}, 9 \mathrm{H}, t-\mathrm{Bu}_{-} \mathrm{CH}_{3}\right), 1.92(\mathrm{~s}$, $3 \mathrm{H}, \mathrm{Ac}-\mathrm{CH}_{3}$ ), 2.21-2.34 (m, $\left.2 \mathrm{H}, \mathrm{H}-4\right), 2.98-3.08$ (m, $2 \mathrm{H}$, $\mathrm{H}-5), 6.18$ (t, $J=7.5 \mathrm{~Hz}, 1 \mathrm{H}, \mathrm{H}-3), 7.09$ ( $\left.\mathrm{s}_{\mathrm{br}}, 1 \mathrm{H}, 2-\mathrm{NH}\right)$, $9.39\left(\mathrm{~s}, 1 \mathrm{H}\right.$, guanidine-NH). $\mathrm{MS}\left(\mathrm{ESI}^{+}\right): \mathrm{m} / z=271.2$ $[\mathrm{M}+\mathrm{H}]^{+}$. HRMS $\left(\mathrm{ESI}^{+}\right)$: calcd. for $\mathrm{C}_{12} \mathrm{H}_{22} \mathrm{~N}_{4} \mathrm{O}_{3}$ $\left([\mathrm{M}+\mathrm{H}]^{+}\right) 271.1765$, found 271.1769 .

$N^{2}$-Acetyl-2,3-didehydro-arginine methyl ester ( $\mathbf{8 d}$ )

General procedure B with (Z)-methyl $N^{2}$-acetyl-2-amino-5azido-pent-2-enoate $(Z)$-11c (204 mg, $0.962 \mathrm{mmol}$ ), $\mathrm{PPh}_{3}$ (504 mg, $1.92 \mathrm{mmol})$, water $(0.4 \mathrm{~mL})$ and THF $(10 \mathrm{~mL})$ for the Staudinger reduction as well as guanidinylation reagent 13 (254 mg, $1.73 \mathrm{mmol}), \mathrm{NEt}_{3} \quad(175 \mathrm{mg}, 240 \mu \mathrm{L}$, $1.73 \mathrm{mmol})$ and DMF $(9 \mathrm{~mL})$ for the guanidinylation reaction. The reaction mixture was stirred at $\mathrm{rt}$ for 2 days. Column chromatography $\left(\mathrm{CH}_{2} \mathrm{Cl}_{2}-\mathrm{MeOH} \quad 10: 1 \rightarrow\right.$ EtOAc$\mathrm{MeOH} 1: 1 \rightarrow \mathrm{MeOH}$ ) gave impure 8d as a yellow oil (71 mg). ${ }^{1} \mathrm{H}$ NMR (300 MHz, DMSO-d 6 ): $\delta=1.93(\mathrm{~s}, 3 \mathrm{H}$, Ac- $\left.\mathrm{CH}_{3}\right), 2.26(\mathrm{td}, J=7.4 \mathrm{~Hz}, 7.1 \mathrm{~Hz}, 2 \mathrm{H}, \mathrm{H}-4), 3.20(\mathrm{dt}$, $J=7.1 \mathrm{~Hz}, 7.0 \mathrm{~Hz}, 2 \mathrm{H}, \mathrm{H}-5), 6.18$ (t, $J=7.4 \mathrm{~Hz}, 1 \mathrm{H}$, $\mathrm{H}-3), 7.58(\mathrm{~s}, 2 \mathrm{H}, 2-\mathrm{NH}), 8.14\left(\mathrm{~s}_{\mathrm{br}}, 3 \mathrm{H}\right.$, guanidine- $\left.\mathrm{NH}\right)$, $9.47\left(\mathrm{~s}, 1 \mathrm{H}\right.$, guanidine-NH). $\mathrm{MS}\left(\mathrm{ESI}^{+}\right): \mathrm{m} / z=229.2$ $[\mathrm{M}+\mathrm{H}]^{+}$. HRMS $\left(\mathrm{ESI}^{+}\right)$: calcd. for $\mathrm{C}_{9} \mathrm{H}_{16} \mathrm{~N}_{4} \mathrm{O}_{3}$ $\left([\mathrm{M}+\mathrm{H}]^{+}\right)$229.1295, found 229.1299.

$N^{2}$-Boc- $N^{8}, N^{9}$-bis-Cbz-2,3-didehydro-arginine tertbutyl ester $(\mathbf{8 e})$

General procedure C with crude $N^{2}$-Boc-2,3-didehydroornithine tert-butyl ester 12 (509 mg, obtained from (Z)-11a as described for the synthesis of ( $\mathrm{rac}$-14,15), guanidinylation reagent 31 ( $828 \mathrm{mg}, 2.31 \mathrm{mmol}), \mathrm{NEt}_{3}$ $(539 \mathrm{mg}, \quad 739 \mu \mathrm{L}, \quad 5.34 \mathrm{mmol}), \quad$ AgOTf $(638 \mathrm{mg}$, $2.49 \mathrm{mmol})$ and DMF $(9 \mathrm{~mL})$. Purification by column chromatography (petroleum ether-EtOAc 6:1 $\rightarrow$ 3:1) gave 8e as a yellow foam $(761 \mathrm{mg}, 71 \%$ over 2 steps from (Z)-11a). ${ }^{1} \mathrm{H}$ NMR (300 MHz, DMSO-d $\left.{ }_{6}\right): \delta=1.39(\mathrm{~s}, 9 \mathrm{H}$, $t$-Bu-CH 3$), 1.40\left(\mathrm{~s}, 9 \mathrm{H}, t-\mathrm{Bu}-\mathrm{CH}_{3}\right), 2.36(\mathrm{dt}, J=7.1 \mathrm{~Hz}$, $6.8 \mathrm{~Hz}, 2 \mathrm{H}, \mathrm{H}-4), 3.42$ (td, $J=6.8 \mathrm{~Hz}, 5.9 \mathrm{~Hz}, 2 \mathrm{H}, \mathrm{H}-5)$, $5.04\left(\mathrm{~s}, 2 \mathrm{H}, \mathrm{Cbz}-\mathrm{CH}_{2}\right), 5.21\left(\mathrm{~s}, 2 \mathrm{H}, \mathrm{Cbz}-\mathrm{CH}_{2}\right), 6.12(\mathrm{t}$, $J=7.1 \mathrm{~Hz}, 1 \mathrm{H}, \mathrm{H}-3), 7.27-7.44(\mathrm{~m}, 10 \mathrm{H}$, Cbz-aryl-H), $8.26\left(\mathrm{~s}_{\mathrm{br}}, 1 \mathrm{H}, 2-\mathrm{NH}\right), 8.47(\mathrm{t}, J=5.9 \mathrm{~Hz}, 1 \mathrm{H}$, guanidine$\mathrm{NH}), 11.59$ (s, $1 \mathrm{H}$, guanidine-NH). ${ }^{13} \mathrm{C}$ NMR $(126 \mathrm{MHz}$, DMSO-d $\left._{6}\right): \delta=26.8(\mathrm{C}-4), 27.5\left(t-\mathrm{Bu}_{-} \mathrm{CH}_{3}\right), 27.9(t-\mathrm{Bu}-$ $\left.\mathrm{CH}_{3}\right), 39.0(\mathrm{C}-5), 66.3\left(\mathrm{Cbz}-\mathrm{CH}_{2}\right), 67.4\left(\mathrm{Cbz}-\mathrm{CH}_{2}\right), 78.5$ $(t$-Bu-C), 79.9 ( $t$-Bu-C), 127.5 (Cbz-aryl-C-2, Cbz-aryl-C-6), 127.7 (Cbz-aryl-C-2, Cbz-aryl-C-6), 128.1 (Cbz-aryl-C-4), 128.2 (Cbz-aryl-C-3, Cbz-aryl-C-5), 128.3 (Cbz-aryl-C-3, Cbz-aryl-C-5), 130.3 (C-3), 134.9 (C-2), 136.6 (Cbz-arylC-1), 152.3 (guanidine-C), 153.2 (Boc-C=O), 154.9 (Cbz$\mathrm{C}=\mathrm{O}), 163.4(\mathrm{C}-1)$. MS $\left(\mathrm{ESI}^{+}\right): \mathrm{m} / z=619.3[\mathrm{M}+\mathrm{Na}]^{+}$. HRMS $\left(\mathrm{ESI}^{+}\right)$: calcd. for $\mathrm{C}_{31} \mathrm{H}_{40} \mathrm{~N}_{4} \mathrm{O}_{8}\left([\mathrm{M}+\mathrm{Na}]^{+}\right)$ 619.2738, found 619.2742. IR (ATR): $v=3324,2977$, 1718, 1638, 1620, 1257, 1204, 1137, 1049, 695. UV $(\mathrm{MeCN}): \lambda_{\max }(\log \varepsilon)=206$ (4.54), 237 (4.41). Mp: $57^{\circ} \mathrm{C}$. TLC: $R_{f}=0.73$ (petroleum ether-EtOAc $1: 1$ ).

$N^{2}$-Boc- $N^{5}$-benzyl- $N^{8}, N^{9}$-bis-Cbz-2,3-didehydroarginine tert-butyl ester (8f)

To a solution of crude $N^{2}$-Boc-2,3-didehydro-ornithine tert-butyl ester $\mathbf{1 2}$ (694 mg, obtained from (Z)-11a as described for the synthesis of $(\mathrm{rac})-\mathbf{1 4}, \mathbf{1 5})$ in $\mathrm{CH}_{2} \mathrm{Cl}_{2}$ $(20 \mathrm{~mL})$, molecular sieves $(4 \AA)$ were added at $\mathrm{rt}$ and the mixture was stirred at $\mathrm{rt}$ for $30 \mathrm{~min}$. Subsequently, benzaldehyde $(259 \mathrm{mg}, 247 \mu \mathrm{L}, 2.45 \mathrm{mmol}$ ) was added, and the reaction mixture was stirred at $\mathrm{rt}$ for $17 \mathrm{~h}$. After filtration, the filtrate was evaporated under reduced pressure. The resultant crude product was dissolved in $\mathrm{MeOH}(20 \mathrm{~mL})$ and cooled to $0{ }^{\circ} \mathrm{C}$. $\mathrm{NaBH}_{4}(229 \mathrm{mg}, 6.07 \mathrm{mmol})$ was added, and the reaction mixture was stirred at $0{ }^{\circ} \mathrm{C}$ for $3 \mathrm{~h}$. The reaction was quenched by addition of saturated $\mathrm{NH}_{4} \mathrm{Cl}$ solution $(5 \mathrm{~mL})$. After addition of water $(10 \mathrm{~mL})$, the aqueous layer was extracted with $\operatorname{EtOAc}(3 \times)$. The combined organics were washed with $\mathrm{H}_{2} \mathrm{O}(25 \mathrm{~mL})$, dried over $\mathrm{Na}_{2} \mathrm{SO}_{4}$ and evaporated under reduced pressure to give 29 as a yellow oil $(750 \mathrm{mg})$. Some of this crude product $(702 \mathrm{mg}$ ) was used without further purification for the guanidinylation reaction according to general procedure $C$ with guanidinylation reagent $\mathbf{3 1}$ (869 mg, $2.43 \mathrm{mmol}$ ), $\mathrm{NEt}_{3}(566 \mathrm{mg}, 775 \mu \mathrm{L}, 5.60 \mathrm{mmol}$ ), AgOTf (669 mg, $2.61 \mathrm{mmol}$ ) and DMF $(9.5 \mathrm{~mL})$. Purification by column chromatography (petroleum ether-EtOAc $4: 1 \rightarrow 1: 1)$ gave $\mathbf{8 f}$ as a white foam $(820 \mathrm{mg}, 55 \%$ over 3 steps from (Z)-11a). ${ }^{1} \mathrm{H}$ NMR (300 MHz, DMSO-d $\mathrm{d}_{6}$ ): $\delta=1.39\left(\mathrm{~s}, 9 \mathrm{H}, t-\mathrm{Bu}-\mathrm{CH}_{3}\right), 1.42\left(\mathrm{~s}, 9 \mathrm{H}, t-\mathrm{Bu}_{-} \mathrm{CH}_{3}\right), 2.39$ (dt, $J=7.4 \mathrm{~Hz}, 7.0 \mathrm{~Hz}, 2 \mathrm{H}, \mathrm{H}-4), 3.42(\mathrm{t}, J=7.0 \mathrm{~Hz}$, $2 \mathrm{H}, \mathrm{H}-5), 4.62$ (s, $\left.2 \mathrm{H}, \mathrm{Bn}-\mathrm{CH}_{2}\right), 4.95$ (s, $2 \mathrm{H}, \mathrm{Cbz}-\mathrm{CH}_{2}$ ), $5.08\left(\mathrm{~s}, 2 \mathrm{H}, \mathrm{Cbz}-\mathrm{CH}_{2}\right), 6.04(\mathrm{t}, J=7.4 \mathrm{~Hz}, 1 \mathrm{H}, \mathrm{H}-3)$, 7.19-7.42 (m, 15 H, aryl-H), $8.28\left(\mathrm{~s}_{\mathrm{br}}, 1 \mathrm{H}, 2-\mathrm{NH}\right), 10.07$ (s, $1 \mathrm{H}$, guanidine-NH). ${ }^{13} \mathrm{C}$ NMR $\left(126 \mathrm{MHz}\right.$, DMSO-d $\left.\mathrm{d}_{6}\right)$ : $\delta=25.4(\mathrm{C}-4), 27.5\left(t-\mathrm{Bu}-\mathrm{CH}_{3}\right), 28.0\left(t-\mathrm{Bu}_{-} \mathrm{CH}_{3}\right), 46.5$

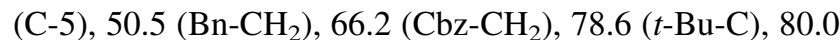
$(t$-Bu-C), 126.9 (aryl-C-2, aryl-C-6), 127.3 (aryl-C-2, aryl- 
C-6), 127.6 (aryl-C-4), 127.8 (aryl-C-4), 128.0 (aryl-C-3, aryl-C-5), 128.2 (aryl-C-3, aryl-C-5), 128.4 (C-2), 130.3 (C-3), 135.8 (aryl-C-1), 136.8 (aryl-C-1), 151.0 (Boc$\mathrm{C}=\mathrm{O}), 152.3$ (guanidine-C), $153.3(\mathrm{Cbz}-\mathrm{C}=\mathrm{O}), 163.3(\mathrm{C}-1)$. MS $\left(\mathrm{ESI}^{+}\right): m / z=709.3[\mathrm{M}+\mathrm{Na}]^{+}$. HRMS $\left(\mathrm{ESI}^{+}\right)$: calcd. for $\mathrm{C}_{38} \mathrm{H}_{46} \mathrm{~N}_{4} \mathrm{O}_{8}\left([\mathrm{M}+\mathrm{Na}]^{+}\right)$709.3208, found 709.3206. TLC: $R_{f}=0.70$ (petroleum ether-EtOAc 1:1).

$N^{2}, N^{2}$-bis-Boc- $N^{8}, N^{9}$-bis-Cbz-2,3-didehydro-arginine tert-butyl ester $(\mathbf{8} \mathbf{g})$

General procedure $\mathrm{C}$ with crude $N^{2}, N^{2}$-bis-Boc-2,3-didehydro-ornithine tert-butyl ester 16 (488 mg, obtained from (Z)-11d as described for the synthesis of (rac)-17,18), guanidinylation reagent 31 (588 mg, $1.64 \mathrm{mmol}^{2}, \mathrm{NEt}_{3}$ (383 mg, $\quad 525 \mu \mathrm{L}, \quad 3.79 \mathrm{mmol})$, AgOTf $(173 \mathrm{mg}$, $0.675 \mathrm{mmol})$ and DMF $(6.5 \mathrm{~mL})$. Purification by column chromatography (petroleum ether-EtOAc 10:1 $\rightarrow$ 1:2) gave $8 \mathrm{~g}$ as a white foam (642 $\mathrm{mg}, 50 \%$ over 2 steps from (Z)-11d). ${ }^{1} \mathrm{H}$ NMR $\left(300 \mathrm{MHz}\right.$, DMSO- $\left.\mathrm{d}_{6}\right): \delta=1.31$ (s, $\left.18 \mathrm{H}, t-\mathrm{Bu}-\mathrm{CH}_{3}\right), 1.38\left(\mathrm{~s}, 9 \mathrm{H}, t-\mathrm{Bu}-\mathrm{CH}_{3}\right), 2.30(\mathrm{dt}$, $J=7.0 \mathrm{~Hz}, 6.6 \mathrm{~Hz}, 2 \mathrm{H}, \mathrm{H}-4), 3.46(\mathrm{td}, J=6.6 \mathrm{~Hz}$, $6.0 \mathrm{~Hz}, 2 \mathrm{H}, \mathrm{H}-5), 5.00$ (s, $\left.2 \mathrm{H}, \mathrm{Cbz}_{-} \mathrm{CH}_{2}\right), 5.18(\mathrm{~s}, 2 \mathrm{H}$, Cbz- $\left.\mathrm{CH}_{2}\right), 6.70(\mathrm{t}, J=7.0 \mathrm{~Hz}, 1 \mathrm{H}, \mathrm{H}-3), 7.24-7.41(\mathrm{~m}$, $10 \mathrm{H}$, Cbz-aryl-H), $8.53(\mathrm{t}, J=6.0 \mathrm{~Hz}, 1 \mathrm{H}$, guanidine$\mathrm{NH}), 11.58\left(\mathrm{~s}, 1 \mathrm{H}\right.$, guanidine-NH). ${ }^{13} \mathrm{C}$ NMR $(76 \mathrm{MHz}$, DMSO-d $\left.\mathrm{d}_{6}\right): \delta=27.2\left(\mathrm{C}-4, t-\mathrm{Bu}-\mathrm{CH}_{3}\right), 27.5\left(t-\mathrm{Bu}-\mathrm{CH}_{3}\right)$, $39.5(\mathrm{C}-5), 66.3\left(\mathrm{Cbz}-\mathrm{CH}_{2}\right), 67.5\left(\mathrm{Cbz}-\mathrm{CH}_{2}\right), 80.6(t$-BuC), 81.7 ( $t$-Bu-C), 127.7 (Cbz-aryl-C-2, Cbz-aryl-C-6), 127.8 (Cbz-aryl-C-2, Cbz-aryl-C-6), 128.0 (Cbz-aryl-C-4), 128.2 (Cbz-aryl-C-4), 128.4 (Cbz-aryl-C-3, Cbz-aryl-C-5), 131.0 (C-2), 135.1 (Cbz-aryl-C-1), 136.8 (Cbz-aryl-C-1), 137.2 (C-3), 149.6 (Boc-C=O), 152.3 (guanidine-C), 155.1 $(\mathrm{Cbz}-\mathrm{C}=\mathrm{O}), 162.0(\mathrm{Cbz}-\mathrm{C}=\mathrm{O}), 162.9(\mathrm{C}-1) . \mathrm{MS}\left(\mathrm{ESI}^{+}\right)$: $m / z=719.4 \quad[\mathrm{M}+\mathrm{Na}]^{+}$. HRMS $\left(\mathrm{ESI}^{+}\right)$: calcd. for $\mathrm{C}_{36} \mathrm{H}_{48} \mathrm{~N}_{4} \mathrm{O}_{10}\left([\mathrm{M}+\mathrm{Na}]^{+}\right)$719.3263, found 719.3274. IR (ATR): $v=2978,1719,1638,1366,1249,1151,1094$, 1047, 734, 696. UV (MeCN): $\lambda_{\max }(\log \varepsilon)=206$ (4.57), 240 (4.45), 273 (4.48). TLC: $R_{f}=0.53$ (petroleum etherEtOAc 1:1).

$N^{2}, N^{2}$-Bis-Boc- $N^{5}$-benzyl- $N^{8}, N^{9}$-bis-Cbz-2,3didehydro-arginine tert-butyl ester $(\mathbf{8} \mathbf{~ h})$

To a solution of crude $N^{2}, N^{2}$-bis-Boc-2,3-didehydro-ornithine tert-butyl ester $\mathbf{1 6}(1.10 \mathrm{~g}$, obtained from $(Z)-\mathbf{1 1 d}$ as described for the synthesis of (rac)-17,18) in $\mathrm{CH}_{2} \mathrm{Cl}_{2}$ $(25 \mathrm{~mL})$, molecular sieves $(4 \AA)$ were added at $\mathrm{rt}$ and the mixture was stirred at rt for $30 \mathrm{~min}$. Subsequently, benzaldehyde (504 mg, $478 \mu \mathrm{L}, 4.75 \mathrm{mmol}$ ) was added, and the reaction mixture was stirred at $\mathrm{rt}$ for $17 \mathrm{~h}$. After filtration, the filtrate was evaporated under reduced pressure. The resultant crude product was dissolved in $\mathrm{MeOH}(25 \mathrm{~mL})$.
$\mathrm{NaBH}_{4}(1.79 \mathrm{~g}, 47.6 \mathrm{mmol})$ was added in two portions (the second after $4 \mathrm{~h}$ ), and the reaction mixture was stirred at $\mathrm{rt}$ for $22 \mathrm{~h}$. The reaction was quenched by addition of saturated $\mathrm{NH}_{4} \mathrm{Cl}$ solution $(5 \mathrm{~mL})$. After addition of water $(10 \mathrm{~mL})$, the aqueous layer was extracted with EtOAc $(3 \times)$. The combined organics were washed with $\mathrm{H}_{2} \mathrm{O}$ $(25 \mathrm{~mL})$, dried over $\mathrm{Na}_{2} \mathrm{SO}_{4}$ and evaporated under reduced pressure. The resultant crude product was separated by column chromatography (petroleum ether-EtOAc 1:1) to give impure $\mathbf{3 0}$ as a yellow oil (748 $\mathrm{mg}$ ). This material was used without further purification for the guanidinylation reaction according to general procedure $\mathrm{C}$ with guanidinylation reagent $31(758 \mathrm{mg}, 2.12 \mathrm{mmol}), \mathrm{NEt}_{3}(493 \mathrm{mg}$, $676 \mu \mathrm{L}, 4.88 \mathrm{mmol})$, AgOTf (584 mg, $2.28 \mathrm{mmol})$ and DMF $(8 \mathrm{~mL})$. Purification by column chromatography (petroleum ether-EtOAc 6:1 $\rightarrow 3: 1$ ) gave $8 \mathbf{h}$ as a yellow foam (991 mg, $42 \%$ over 3 steps from (Z)-11d). ${ }^{1} \mathrm{H}$ NMR (300 MHz, DMSO-d ${ }_{6}$ ): $\delta=1.32\left(\mathrm{~s}, 18 \mathrm{H}, t-\mathrm{Bu}^{-} \mathrm{CH}_{3}\right), 1.39$ $(\mathrm{s}, 9 \mathrm{H}, t$-Bu-CH$), 2.27(\mathrm{dt}, J=7.6 \mathrm{~Hz}, 7.1 \mathrm{~Hz}, 2 \mathrm{H}$, $\mathrm{H}-4), 3.42(\mathrm{t}, J=7.1 \mathrm{~Hz}, 2 \mathrm{H}, \mathrm{H}-5), 4.62(\mathrm{~s}, 2 \mathrm{H}$, $\left.\mathrm{Bn}-\mathrm{CH}_{2}\right), 4.90\left(\mathrm{~s}, 2 \mathrm{H}, \mathrm{Cbz}-\mathrm{CH}_{2}\right), 5.03\left(\mathrm{~s}, 2 \mathrm{H}, \mathrm{Cbz}-\mathrm{CH}_{2}\right)$, $6.59(\mathrm{t}, J=7.6 \mathrm{~Hz}, 1 \mathrm{H}, \mathrm{H}-3), 7.17-7.33(\mathrm{~m}, 15 \mathrm{H}$, aryl$\mathrm{H}), 10.05$ (s, $1 \mathrm{H}, \mathrm{NH}) .{ }^{13} \mathrm{C} \mathrm{NMR}\left(126 \mathrm{MHz}, \mathrm{DMSO}-\mathrm{d}_{6}\right)$ : $\delta=25.9(\mathrm{C}-4), 27.3\left(t-\mathrm{Bu}-\mathrm{CH}_{3}\right), 27.5\left(t-\mathrm{Bu}_{-} \mathrm{CH}_{3}\right), 45.8$

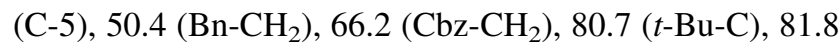
$(t-\mathrm{Bu}-\mathrm{C}), 126.2$ (aryl-C-2, aryl-C-6), 126.9 (aryl-C-2, arylC-6), 127.6 (aryl-C-4), 127.8 (aryl-C-4), 128.0 (aryl-C-3, aryl-C-5), 128.3 (aryl-C-3, aryl-C-5), 130.9 (C-2), 136.0 (aryl-C-1), 136.6 (C-3) 136.8 (aryl-C-1), 149.5 (Boc-C=O), 152.3 (guanidine-C), $153.3(\mathrm{Cbz}-\mathrm{C}=\mathrm{O}), 161.8(\mathrm{C}-1)$. MS $\left(\mathrm{ESI}^{+}\right): m / z=809.4[\mathrm{M}+\mathrm{Na}]^{+}$. HRMS $\left(\mathrm{ESI}^{+}\right)$: calcd. for $\mathrm{C}_{43} \mathrm{H}_{54} \mathrm{~N}_{4} \mathrm{O}_{10}\left([\mathrm{M}+\mathrm{Na}]^{+}\right)$809.3732, found 809.3736. IR (ATR): $v=2977,1718,1602,1454,1366,1250,1150$, 1090, 733, 696. UV (MeCN): $\lambda_{\max }(\log \varepsilon)=207$ (4.61), 251 (4.26). TLC: $R_{f}=0.71$ (petroleum ether-EtOAc 1:1).

tert-Butyl $N^{2}$-Boc-2-amino-5-azido-pent-2-enoate (11a)

General procedure A with phosphonate 9a (2.00 g, $5.45 \mathrm{mmol})$, crude 3-azido-propionaldehyde $\mathbf{1 0}$ (1.18 g), $\mathrm{KO} t$-Bu (641 mg, $5.72 \mathrm{mmol})$ and THF (11 mL (KOt-Bu), $16 \mathrm{~mL}(\mathbf{9 a}), 16 \mathrm{~mL}(\mathbf{1 0}))$. Purification by column chromatography (petroleum ether-EtOAc 6:1) gave (Z)-11a as a colorless solid $(1.31 \mathrm{~g}, 77 \%)$ and $(E)$-11a as a colorless oil (62 mg, $4 \%$ ). (Z)-11a: ${ }^{1} \mathrm{H}$ NMR $\left(300 \mathrm{MHz}, \mathrm{CDCl}_{3}\right)$ : $\delta=1.44\left(\mathrm{~s}, 9 \mathrm{H}, t-\mathrm{Bu}-\mathrm{CH}_{3}\right), 1.47\left(\mathrm{~s}, 9 \mathrm{H}, t-\mathrm{Bu}_{-} \mathrm{CH}_{3}\right), 2.45$ $(\mathrm{dt}, J=7.4 \mathrm{~Hz}, 6.9 \mathrm{~Hz}, 2 \mathrm{H}, \mathrm{H}-4), 3.41(\mathrm{t}, J=6.9 \mathrm{~Hz}$, $2 \mathrm{H}, \mathrm{H}-5), 6.19\left(\mathrm{~s}_{\mathrm{br}}, 1 \mathrm{H}, \mathrm{NH}\right), 6.38(\mathrm{t}, J=7.4 \mathrm{~Hz}, 1 \mathrm{H}$, $\mathrm{H}-3) .{ }^{13} \mathrm{C}$ NMR $\left(76 \mathrm{MHz}, \mathrm{CDCl}_{3}\right): \delta=28.0\left(t-\mathrm{Bu}_{-} \mathrm{CH}_{3}\right)$, $28.2\left(t-\mathrm{Bu}-\mathrm{CH}_{3}\right), 28.5(\mathrm{C}-4), 50.0(\mathrm{C}-5), 80.5(t-\mathrm{Bu}-\mathrm{C})$, $82.0(t$-Bu-C), $128.5(\mathrm{C}-3), 128.7(\mathrm{C}-2), 153.1(\mathrm{Boc}-\mathrm{C}=\mathrm{O})$, $163.6(\mathrm{C}-1)$. MS $\left(\mathrm{ESI}^{+}\right): m / z=335[\mathrm{M}+\mathrm{Na}]^{+}$. HRMS $\left(\mathrm{ESI}^{+}\right)$: calcd. for $\mathrm{C}_{14} \mathrm{H}_{24} \mathrm{~N}_{4} \mathrm{O}_{4}\left([\mathrm{M}+\mathrm{Na}]^{+}\right) 335.1690$, 
found 335.1690. IR: $v=3308,2980,2097,1688,1493$, 1367, 1246, 1146, 847, 778. UV (MeCN): $\lambda_{\max }$ $(\log \varepsilon)=231$ (3.85). Mp: $57{ }^{\circ} \mathrm{C}$. TLC: $R_{f}=0.42$ (petroleum ether-EtOAc 3:1). (E)-11a: ${ }^{1} \mathrm{H}$ NMR $(300 \mathrm{MHz}$, $\left.\mathrm{CDCl}_{3}\right): \delta=1.44\left(\mathrm{~s}, 9 \mathrm{H}, t-\mathrm{Bu}-\mathrm{CH}_{3}\right), 1.52(\mathrm{~s}, 9 \mathrm{H}, t-\mathrm{Bu}-$ $\mathrm{CH}_{3}$ ), 2.79 (dt, $\left.J=7.5 \mathrm{~Hz}, 7.3 \mathrm{~Hz}, 2 \mathrm{H}, \mathrm{H}-4\right), 3.35(\mathrm{t}$, $J=7.4 \mathrm{~Hz}, 2 \mathrm{H}, \mathrm{H}-5), 6.66(\mathrm{t}, J=7.5 \mathrm{~Hz}, 1 \mathrm{H}, \mathrm{H}-3)$, $6.74\left(\mathrm{~s}_{\mathrm{br}}, 1 \mathrm{H}, \mathrm{NH}\right) .{ }^{13} \mathrm{C} \mathrm{NMR}\left(76 \mathrm{MHz} \mathrm{CDCl}_{3}\right): \delta=28.0$ $(\mathrm{C}-4), 28.1\left(t-\mathrm{Bu}-\mathrm{CH}_{3}\right), 28.3\left(t-\mathrm{Bu}_{-} \mathrm{CH}_{3}\right), 51.0(\mathrm{C}-5), 80.3$ $(t$-Bu-C), 83.3 ( $t$-Bu-C), 121.3 (C-3), 128.0 (C-2), 153.0 $(\mathrm{Boc}-\mathrm{C}=\mathrm{O}), \quad 163.1 \quad(\mathrm{C}-1) . \quad \mathrm{MS} \quad\left(\mathrm{ESI}^{+}\right): \quad \mathrm{m} / z=335$ $[\mathrm{M}+\mathrm{Na}]^{+}$. HRMS $\left(\mathrm{ESI}^{+}\right)$: calcd. for $\mathrm{C}_{14} \mathrm{H}_{24} \mathrm{~N}_{4} \mathrm{O}_{4}$ $\left([\mathrm{M}+\mathrm{Na}]^{+}\right)$335.1690, found 335.1690. TLC: $R_{f}=0.42$ (petroleum ether-EtOAc 3:1).

tert-Butyl $N^{2}$-acetyl-2-amino-5-azido-pent-2-enoate (11b)

General procedure A with phosphonate 9b (1.64 g, $5.83 \mathrm{mmol}$ ), crude 3-azido-propionaldehyde 10 (1.15 g), $\mathrm{KO} t-\mathrm{Bu}(686 \mathrm{mg}, 6.12 \mathrm{mmol})$ and THF (11 mL (KOt-Bu), $16 \mathrm{~mL}$ (9b), $10 \mathrm{~mL}(\mathbf{1 0})$ ). Purification by column chromatography (petroleum ether-EtOAc 3:2) gave $(Z)-11 b$ as a yellow oil $(910 \mathrm{mg}, 61 \%)$ while $(E)$-11b could not be isolated. ${ }^{1} \mathrm{H} \mathrm{NMR}\left(300 \mathrm{MHz}, \mathrm{CDCl}_{3}\right): \delta=1.48(\mathrm{~s}, 9 \mathrm{H}$, $t$-Bu-CH$\left.{ }_{3}\right), 2.10\left(\mathrm{~s}, 3 \mathrm{H}, \mathrm{Ac}-\mathrm{CH}_{3}\right), 2.40(\mathrm{dt}, J=7.1 \mathrm{~Hz}$, $J=6.7 \mathrm{~Hz}, 2 \mathrm{H}, \mathrm{H}-4), 3.43(\mathrm{t}, J=6.7 \mathrm{~Hz}, 2 \mathrm{H}, \mathrm{H}-5)$, $6.49(\mathrm{t}, J=7.1 \mathrm{~Hz}, 1 \mathrm{H}, \mathrm{H}-3), 7.13\left(\mathrm{~s}_{\mathrm{br}}, 1 \mathrm{H}, \mathrm{NH}\right)$.

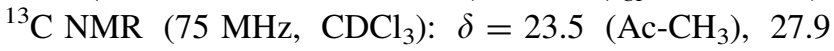
$\left(t-\mathrm{Bu}-\mathrm{CH}_{3}\right), 29.0(\mathrm{C}-4), 49.9$ (C-5), 82.3 ( $t$-Bu-C), 127.6 (C-2), 130.8 (C-3), 163.4 (C-1), 168.2 (Ac-C=O). MS: $\left(\mathrm{ESI}^{+}\right): m / z=531.3[2 \mathrm{M}+\mathrm{Na}]^{+}$. HRMS $\left(\mathrm{ESI}^{+}\right)$: calcd. for $\mathrm{C}_{11} \mathrm{H}_{18} \mathrm{~N}_{4} \mathrm{O}_{3}\left([\mathrm{M}-\mathrm{H}]^{-}\right)$253.1306, found 253.1312. IR (ATR): $v=3265,2979,2095,1656,1509,1366,1254$, 1135, 848, 735. UV $(\mathrm{MeCN}): \lambda_{\max }(\log \varepsilon)=230$ (3.81). TLC: $R_{f}=0.34$ (petroleum ether-EtOAc 3:2).

\section{Methyl $N^{2}$-acetyl-2-amino-5-azido-pent-2-enoate (11c)}

General procedure A with phosphonate 9c (1.62 g, $6.80 \mathrm{mmol}$ ), crude 3-azido-propionaldehyde 10 (1.50 g), $\mathrm{KO} t$-Bu (799 mg, $7.14 \mathrm{mmol})$ and THF (14 mL (KOt-Bu), $15 \mathrm{~mL}$ (9c), $10 \mathrm{~mL}(\mathbf{1 0}))$. Purification by column chromatography (petroleum ether-EtOAc 1:1 $\rightarrow$ 1:3) gave (Z)-11c as a yellow solid (751 mg, $52 \%)$ while $(E)$-11c could not be isolated. ${ }^{1} \mathrm{H}-\mathrm{NMR}\left(300 \mathrm{MHz}, \mathrm{CDCl}_{3}\right)$ : $\delta=2.13\left(\mathrm{~s}, 3 \mathrm{H}, \mathrm{Ac}-\mathrm{CH}_{3}\right), 2.43(\mathrm{dt}, J=7.1 \mathrm{~Hz}, 6.7 \mathrm{~Hz}$, $2 \mathrm{H}, \mathrm{H}-4), 3.46$ (t, $J=6.7 \mathrm{~Hz} 2 \mathrm{H}, \mathrm{H}-5), 3.78(\mathrm{~s}, 3 \mathrm{H}$, $\left.\mathrm{OCH}_{3}\right), 6.64(\mathrm{t}, J=7.1 \mathrm{~Hz}, 1 \mathrm{H}, \mathrm{H}-3), 7.09\left(\mathrm{~s}_{\mathrm{br}}, 1 \mathrm{H}, \mathrm{NH}\right)$.

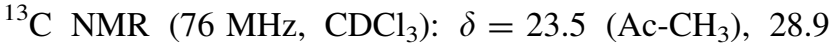
(C-4), 49.8 (C-5), $52.6\left(\mathrm{OCH}_{3}\right), 126.5(\mathrm{C}-2), 132.7$ (C-3), 164.8 (C-1), 168.3 (Ac-C=O). $\mathrm{MS}\left(\mathrm{ESI}^{+}\right): \mathrm{m} / z=447.2$
$[2 \mathrm{M}+\mathrm{Na}]^{+}$. HRMS $\left(\mathrm{ESI}^{+}\right)$: calcd. for $\mathrm{C}_{8} \mathrm{H}_{12} \mathrm{~N}_{4} \mathrm{O}_{3}$ $\left([\mathrm{M}+\mathrm{Na}]^{+}\right)$235.0802, found 235.0803. IR (ATR): $v=3261,2962,2086,1742,1676,1372,1210,1021,795$, 714. UV $(\mathrm{MeCN}): \lambda_{\max }(\log \varepsilon)=231$ (3.80). Mp: $51{ }^{\circ} \mathrm{C}$. TLC: $R_{f}=0.16$ (petroleum ether-EtOAc 1:1).

tert-Butyl $N^{2}, N^{2}$-bis-Boc-2-amino-5-azido-pent-2enoate (11d)

To a solution of (Z)-tert-butyl $N^{2}$-Boc-2-amino-5-azidopent-2-enoate $(Z)-11 \mathrm{a}(1.29 \mathrm{~g}, 4.14 \mathrm{mmol})$ in $\mathrm{MeCN}$ $12 \mathrm{~mL}$, DMAP (50 mg, $0.41 \mathrm{mmol}$ ) and $\mathrm{Boc}_{2} \mathrm{O}(1.98 \mathrm{~g}$, $9.10 \mathrm{mmol}$ ) were added at $\mathrm{rt}$. The reaction mixture was stirred at $\mathrm{rt}$ for $20 \mathrm{~h}$. The solvent was evaporated under reduced pressure. After the addition of EtOAc, the solution was washed with aqueous $\mathrm{KHSO}_{4}$ solution (1 M, $2 \times 10 \mathrm{~mL})$, saturated $\mathrm{NaHCO}_{3}$ solution $(1 \times 10 \mathrm{~mL})$ and brine $(1 \times 10 \mathrm{~mL})$, dried over $\mathrm{Na}_{2} \mathrm{SO}_{4}$ and evaporated under reduced pressure. The resultant crude product was purified by column chromatography (petroleum etherEtOAc 10:1) to give (Z)-11d as a colorless oil $(1.57 \mathrm{~g}$, $92 \%) .{ }^{1} \mathrm{H}$ NMR (300 MHz, DMSO-d $\left.{ }_{6}\right): \delta=1.37$ (s, $\left.18 \mathrm{H}, t-\mathrm{Bu}-\mathrm{CH}_{3}\right), 1.41\left(\mathrm{~s}, 9 \mathrm{H}, t-\mathrm{Bu}-\mathrm{CH}_{3}\right), 2.29$ (dt, $J=7.3 \mathrm{~Hz}, 6.6 \mathrm{~Hz}, 2 \mathrm{H}, \mathrm{H}-4), 3.44(\mathrm{t}, J=6.6 \mathrm{~Hz}, 2 \mathrm{H}$, $\mathrm{H}-5), 6.65$ (t, $J=7.3 \mathrm{~Hz}, 1 \mathrm{H}, \mathrm{H}-3) .{ }^{13} \mathrm{C} \mathrm{NMR}(76 \mathrm{MHz}$, DMSO-d $\left.{ }_{6}\right): \delta=27.0(\mathrm{C}-4), 27.3\left(t-\mathrm{Bu}_{-} \mathrm{CH}_{3}\right), 27.5(t-\mathrm{Bu}-$ $\left.\mathrm{CH}_{3}\right), 48.6$ (C-5), 80.8 (t-Bu-C), 81.9 ( $t$-Bu-C), 131.5 (C-2), 136.3 (C-3), 149.7 (Boc-C=O), 161.9 (C-1). MS $\left(\mathrm{ESI}^{+}\right): m / z=847.6[2 \mathrm{M}+\mathrm{Na}]^{+}$. HRMS $\left(\mathrm{ESI}^{+}\right)$: calcd. for $\mathrm{C}_{19} \mathrm{H}_{32} \mathrm{~N}_{4} \mathrm{O}_{6}\left([\mathrm{M}+\mathrm{Na}]^{+}\right)$435.2214, found 435.2218. IR (ATR): $v=2979,2097,1795,1717,1366,1273,1250$, 1150, 1092, 849. UV $(\mathrm{MeCN}): \lambda_{\max }(\log \varepsilon)=216(4.01)$. TLC: $R_{f}=0.23$ (petroleum ether-EtOAc 10:1).

$N^{2}$-Boc-capreomycidine tert-butyl ester ( $(r a c)$-14) and $N^{2}$-Boc-epicapreomycidine tert-butyl ester ((rac)-15)

General procedure B with (Z)-tert-butyl $N^{2}$-Boc-2-amino5-azido-pent-2-enoate (Z)-11a (1.13 g, $3.61 \mathrm{mmol}), \mathrm{PPh}_{3}$ (2.28 g, $8.71 \mathrm{mmol})$, water $(1.7 \mathrm{~mL})$ and THF $(44 \mathrm{~mL})$ for the Staudinger reduction as well as guanidinylation reagent 13 (964 mg, $6.58 \mathrm{mmol}), \mathrm{NEt}_{3} \quad(1.66 \mathrm{~g}, 2.28 \mathrm{~mL}$, $16.4 \mathrm{mmol})$ and DMF $(17 \mathrm{~mL})$ for the domino reaction. The reaction mixture was stirred at $\mathrm{rt}$ for $20 \mathrm{~h}$ and at $70{ }^{\circ} \mathrm{C}$ for $7 \mathrm{~h}$. Purification by column chromatography $\left(\mathrm{CH}_{2} \mathrm{Cl}_{2}-\right.$ $\mathrm{MeOH}$ gradient (3-10\%)) gave a mixture of (rac)-14 and (rac)-15 (ratio $1.0: 1.0$ ) as a yellow foam $(580 \mathrm{mg}, 54 \%$ over 2 steps from (Z)-11a). ${ }^{1} \mathrm{H}$ NMR (300 MHz, $\left.\mathrm{CDCl}_{3}\right)$ : $\delta=1.40\left(\mathrm{~s}, 1 \times 9 \mathrm{H}, t-\mathrm{Bu}-\mathrm{CH}_{3}\right), 1.41(\mathrm{~s}, 1 \times 9 \mathrm{H}, t-\mathrm{Bu}-$ $\left.\mathrm{CH}_{3}\right), 1.46\left(\mathrm{~s}, 1 \times 9 \mathrm{H}, t-\mathrm{Bu}-\mathrm{CH}_{3}\right), 1.47(\mathrm{~s}, 1 \times 9 \mathrm{H}, t-\mathrm{Bu}-$ $\left.\mathrm{CH}_{3}\right)$, 1.79-1.93 (m, $\left.1 \times 2 \mathrm{H}, \mathrm{H}-4\right), 1.93-2.03(\mathrm{~m}$, 
$1 \times 2 \mathrm{H}, \mathrm{H}-4), 3.21-3.33(\mathrm{~m}, 1 \times 2 \mathrm{H}, \mathrm{H}-5), 3.33-3.47$ $(\mathrm{m}, \quad 1 \times 2 \mathrm{H}, \quad \mathrm{H}-5), \quad 3.76-3.83(\mathrm{~m}, 1 \times 1 \mathrm{H}, \mathrm{H}-3)$, 3.85-3.93 (m, $1 \times 1 \mathrm{H}, \mathrm{H}-3), 4.25-4.35(\mathrm{~m}, 2 \times 1 \mathrm{H}$, $\mathrm{H}-2), 5.71\left(\mathrm{~d}_{\mathrm{br}}, J=8.2 \mathrm{~Hz}, 2 \times 1 \mathrm{H}, \mathrm{NH}-2\right), 7.04\left(\mathrm{~s}_{\mathrm{br}}\right.$, $1 \times 2 \mathrm{H}$, guanidine- $\mathrm{NH}), 7.15\left(\mathrm{~s}_{\mathrm{br}}, 1 \times 2 \mathrm{H}\right.$, guanidine$\mathrm{NH}), \quad 7.82\left(\mathrm{~s}_{\mathrm{br}}, \quad 2 \times 1 \mathrm{H}\right.$, guanidine- $\left.\mathrm{NH}\right), 8.10\left(\mathrm{~s}_{\mathrm{br}}\right.$, $1 \times 1 \mathrm{H}$, guanidine- $\mathrm{NH}), 8.18\left(\mathrm{~s}_{\mathrm{br}}, 1 \times 1 \mathrm{H}\right.$, guanidine$\mathrm{NH}) .{ }^{13} \mathrm{C}$ NMR $\left(76 \mathrm{MHz}, \mathrm{CDCl}_{3}\right): \delta=21.7(1 \times \mathrm{C}-4)$, $23.1(1 \times \mathrm{C}-4), 28.0\left(2 \times t-\mathrm{Bu}_{-} \mathrm{CH}_{3}\right), 28.2(1 \times t$-Bu$\left.\mathrm{CH}_{3}\right), 28.3\left(1 \times t-\mathrm{Bu}-\mathrm{CH}_{3}\right), 36.6(1 \times \mathrm{C}-5), 36.7(1 \times$ $\mathrm{C}-5), 50.1(1 \times \mathrm{C}-3), 51.6(1 \times \mathrm{C}-3), 56.4(1 \times \mathrm{C}-2), 56.9$ $(1 \times \mathrm{C}-2), 80.4(1 \times t$-Bu-C $), 80.7(1 \times t$-Bu-C $), 83.6$ $(1 \times t$-Bu-C $), 83.7(1 \times t$-Bu-C $), 154.9(1 \times$ Boc-C $=\mathrm{O})$, $155.1(1 \times$ Boc- $\mathrm{C}=\mathrm{O}), \quad 155.8(1 \times$ guanidine-C $), \quad 156.0$ $(1 \times$ guanidine-C), $168.0(1 \times \mathrm{C}-1), 168.7(1 \times \mathrm{C}-1)$. MS $\left(\mathrm{ESI}^{+}\right): m / z=329.3[\mathrm{M}+\mathrm{H}]^{+}$. HRMS $\left(\mathrm{ESI}^{+}\right)$calcd. for $\mathrm{C}_{15} \mathrm{H}_{28} \mathrm{~N}_{4} \mathrm{O}_{4}\left([\mathrm{M}+\mathrm{H}]^{+}\right)$329.2183, found 329.2183. TLC: $R_{f}=0.33\left(\mathrm{CH}_{2} \mathrm{Cl}_{2}-\mathrm{MeOH}\right.$ 9:1).

$N^{2}, N^{2}$-Bis-Boc-capreomycidine tert-butyl ester $((r a c)-17)$ and $N^{2}, N^{2}$-Bis-Boc-epicapreomycidine tert-butyl ester ((rac)-18)

General procedure B with (Z)-tert-butyl $N^{2}, N^{2}$-bis-Boc-2amino-5-azido-pent-2-enoate (Z)-11d $\quad(671 \mathrm{mg}, \quad 1.63$ $\mathrm{mmol}), \mathrm{PPh}_{3}(1.32 \mathrm{~g}, 5.05 \mathrm{mmol})$, water $(1.0 \mathrm{~mL})$ and THF $(25 \mathrm{~mL})$ for the Staudinger reduction as well as guanidinylation reagent 13 (388 mg, $2.65 \mathrm{mmol}$ ), $\mathrm{NEt}_{3}$ (268 mg, $367 \mu \mathrm{L}, 2.65 \mathrm{mmol}$ ) and DMF $(13 \mathrm{~mL}$ ) for the domino reaction. The reaction mixture was stirred at $\mathrm{rt}$ for $4 \mathrm{~d}$. Purification by column chromatography $\left(\mathrm{CH}_{2} \mathrm{Cl}_{2}-\right.$ $\mathrm{MeOH}$ gradient (5-10\%)) gave a mixture of $(\mathrm{rac})-\mathbf{1 7}$ and (rac)-18 (ratio 1.8:1.0) as a yellow foam $(340 \mathrm{mg}, 49 \%$ over 2 steps from (Z)-11d). ${ }^{1} \mathrm{H}$ NMR (300 MHz, DMSO$\left.\mathrm{d}_{6}\right): \delta=1.39\left(\mathrm{~s}, 2 \times 18 \mathrm{H}, t-\mathrm{Bu}-\mathrm{CH}_{3}\right), 1.40(\mathrm{~s}, 1 \times 9 \mathrm{H}$, $t$-Bu-CH$\left.{ }_{3}\right), 1.45\left(\mathrm{~s}, 1 \times 9 \mathrm{H}, t-\mathrm{Bu}-\mathrm{CH}_{3}\right), 1.96-2.02(\mathrm{~m}$, $2 \times 2 \mathrm{H}, \mathrm{H}-4), 3.21-3.28(\mathrm{~m}, 2 \times 2 \mathrm{H}, \mathrm{H}-5), 3.98-4.07$ $(\mathrm{m}, 2 \times 1 \mathrm{H}, \mathrm{H}-3), 4.61(\mathrm{~d}, J=8.9 \mathrm{~Hz}, 1 \times 1 \mathrm{H}, \mathrm{H}-2)$, $4.70(\mathrm{~d}, J=8.3 \mathrm{~Hz}, 1 \times 1 \mathrm{H}, \mathrm{H}-2), 7.01(\mathrm{~s}, 1 \times 2 \mathrm{H}$, guanidine-NH), $7.09(\mathrm{~s}, 1 \times 2 \mathrm{H}$, guanidine- $\mathrm{NH}), 7.51\left(\mathrm{~s}_{\mathrm{br}}\right.$, $1 \times 1 \mathrm{H}$, guanidine- $\mathrm{NH}), 7.56\left(\mathrm{~s}_{\mathrm{br}}, 1 \times 1 \mathrm{H}\right.$, guanidine$\mathrm{NH}), \quad 8.13\left(\mathrm{~s}_{\mathrm{br}}, \quad 1 \times 1 \mathrm{H}, \quad\right.$ guanidine-NH $), 8.19\left(\mathrm{~s}_{\mathrm{br}}\right.$, $1 \times 1 \mathrm{H}$, guanidine-NH). ${ }^{13} \mathrm{C}$ NMR $\left(76 \mathrm{MHz}\right.$, DMSO-d $\left.\mathrm{d}_{6}\right)$ : $\delta=22.8(2 \times \mathrm{C}-4), 27.4\left(2 \times t-\mathrm{Bu}_{-}-\mathrm{CH}_{3}\right), 27.5(2 \times$ $t$-Bu-CH $\left.\mathrm{CH}_{3}\right), 27.5(2 \times t$-Bu-CH 3$), 34.2(1 \times \mathrm{C}-5), \quad 35.0$ $(1 \times \mathrm{C}-5), 46.8(1 \times \mathrm{C}-3), 47.3(1 \times \mathrm{C}-3), 60.0(1 \times$ $\mathrm{C}-2), 60.3(1 \times \mathrm{C}-2), 81.6(1 \times t$-Bu-C $), 82.0(1 \times t$-BuC), $\quad 83.0 \quad(1 \times t$-Bu-C $), \quad 83.4 \quad(1 \times t$-Bu-C $), \quad 151.8$ $(1 \times$ Boc-C $=0), 151.8(1 \times$ Boc-C$=O), 153.6(2 \times$ guanidine-C $), 154.0(2 \times$ Boc-C=O $), 166.9(2 \times \mathrm{C}-1)$. MS $\left(\mathrm{ESI}^{+}\right): m / z=429.3[\mathrm{M}+\mathrm{H}]^{+}$. HRMS $\left(\mathrm{ESI}^{+}\right)$: calcd. for $\mathrm{C}_{20} \mathrm{H}_{36} \mathrm{~N}_{4} \mathrm{O}_{6}\left([\mathrm{M}+\mathrm{H}]^{+}\right)$429.2708, found 429.2707. TLC: $R_{f}=0.45\left(\mathrm{CH}_{2} \mathrm{Cl}_{2}-\mathrm{MeOH} 9: 1\right)$.
tert-Butyl $N^{2}$-Cbz-2-amino-5-azido-pent-2-enoate (21)

General procedure A with phosphonate 20 (3.46 g, $9.27 \mathrm{mmol})$, crude 3-azido-propionaldehyde $\mathbf{1 0}(1.84 \mathrm{~g})$, $\mathrm{KO} t$-Bu (1.09 g, $9.73 \mathrm{mmol})$ and THF (19 mL (KOt-Bu), $28 \mathrm{~mL}(\mathbf{2 0}), 28 \mathrm{~mL}(\mathbf{1 0}))$. Purification by column chromatography (petroleum ether-EtOAc 8:1) gave (Z)-21 (containing traces of aldehyde 10) as a colorless oil (2.70 g, $81 \%$ as calculated from the ${ }^{1} \mathrm{H}$ NMR spectrum) and $(E)-\mathbf{2 1}$ as a colorless oil $(118 \mathrm{mg}, 3 \%)$. $(Z)-\mathbf{2 1}$ : ${ }^{1} \mathrm{H}$ NMR $\left(300 \mathrm{MHz}, \mathrm{CDCl}_{3}\right): \delta=1.49\left(\mathrm{~s}, 9 \mathrm{H}, t-\mathrm{Bu}_{-} \mathrm{CH}_{3}\right)$, $2.48(\mathrm{dt}, J=7.2 \mathrm{~Hz}, \quad 6.8 \mathrm{~Hz}, 2 \mathrm{H}, \mathrm{H}-4), 3.44(\mathrm{t}$, $J=6.8 \mathrm{~Hz} 2 \mathrm{H}, \mathrm{H}-5), 5.14\left(\mathrm{~s}, 2 \mathrm{H}, \mathrm{Cbz}-\mathrm{CH}_{2}\right), 6.45\left(\mathrm{t}_{\mathrm{br}}\right.$, $J=7.2 \mathrm{~Hz}, 2 \mathrm{H}, \mathrm{H}-3, \mathrm{NH}), 7.30-7.39$ (m, $5 \mathrm{H}$, Cbz-arylH). ${ }^{13} \mathrm{C}$ NMR $\left(76 \mathrm{MHz}, \mathrm{CDCl}_{3}\right): \delta=28.0\left(t-\mathrm{Bu}_{-} \mathrm{CH}_{3}\right)$, 28.5 (C-4), 49.9 (C-5), $67.4\left(\mathrm{Cbz}-\mathrm{CH}_{2}\right), 82.3(t$-Bu-C), 128.2 (Cbz-aryl-C-2, Cbz-aryl-C-6), 128.3 (Cbz-aryl-C-4), 128.5 (C-2, Cbz-aryl-C-3, Cbz-aryl-C-5), 129.7 (C-3), 135.9 (Cbz-aryl-C-1), 153.9 (Cbz-C=O), $163.2(\mathrm{C}-1)$. MS $\left(\mathrm{ESI}^{+}\right): \mathrm{m} / \mathrm{z}=715.3[2 \mathrm{M}+\mathrm{Na}]^{+}$. HRMS $\left(\mathrm{ESI}^{+}\right)$: calcd. for $\mathrm{C}_{17} \mathrm{H}_{22} \mathrm{~N}_{4} \mathrm{O}_{4}\left([\mathrm{M}+\mathrm{Na}]^{+}\right)$369.1533, found 369.1530 . IR (ATR): $v=2971,2095,1703,1498,1290,1218,1147$, 1022, 750, 696. UV $(\mathrm{MeCN}): \lambda_{\mathrm{ma}} \times(\log \varepsilon)=204(4.13)$, 228 (3.83). TLC: $R_{f}=0.73$ (petroleum ether-EtOAc 1:1). (E)-21: ${ }^{1} \mathrm{H}$ NMR $\left(300 \mathrm{MHz}, \mathrm{CDCl}_{3}\right): \delta=1.52(\mathrm{~s}, 9 \mathrm{H}$, $t$-Bu-CH 3 ), 2.82 (dt, $J=7.5 \mathrm{~Hz}, 7.2 \mathrm{~Hz}, 2 \mathrm{H}, \mathrm{H}-4), 3.36$ (t, $J=7.2 \mathrm{~Hz} 2 \mathrm{H}, \mathrm{H}-5), 5.11\left(\mathrm{~s}, 2 \mathrm{H}, \mathrm{Cbz}-\mathrm{CH}_{2}\right), 6.76(\mathrm{t}$, $J=7.5 \mathrm{~Hz}, 1 \mathrm{H}, \mathrm{H}-3), 6.98\left(\mathrm{~s}_{\mathrm{br}}, 1 \mathrm{H}, \mathrm{NH}\right), 7.27-7.39$ (m, $5 \mathrm{H}$, Cbz-aryl-H). ${ }^{13} \mathrm{C}$ NMR $\left(76 \mathrm{MHz}, \mathrm{CDCl}_{3}\right): \delta=28.0$ (C-4), $28.1\left(t-\mathrm{Bu}_{-} \mathrm{CH}_{3}\right), 50.9(\mathrm{C}-5), 66.8\left(\mathrm{Cbz}-\mathrm{CH}_{2}\right), 83.6$ ( $t$-Bu-C), 122.2 (C-3), 127.6 (C-2), 128.2 (Cbz-aryl-C-2, Cbz-aryl-C-6), 128.3 (Cbz-aryl-C-4), 128.6 (Cbz-aryl-C-3, Cbz-aryl-C-5), 136.0 (Cbz-aryl-C-1), $153.5(\mathrm{Cbz}-\mathrm{C}=\mathrm{O})$, $162.7(\mathrm{C}-1)$. MS $\left(\mathrm{ESI}^{+}\right): m / z=715.3\left[2 \mathrm{M}+\mathrm{Na}^{+}\right.$. HRMS (ESI): calcd. for $\mathrm{C}_{17} \mathrm{H}_{22} \mathrm{~N}_{4} \mathrm{O}_{4}\left([\mathrm{M}+\mathrm{Na}]^{+}\right)$ 369.1533, found 369.1535. IR (ATR): $v=2972,2095$, $1697,1509,1363,1215,1151,1046,846,696$. UV $(\mathrm{MeCN}): \quad \lambda_{\max }(\log \varepsilon)=238 \quad(3.85) . \quad$ TLC: $\quad R_{f}=0.78$ (petroleum ether-EtOAc 1:1).

$N^{2}$-Cbz-capreomycidine tert-butyl ester ( $(r a c)$-23) and $N^{2}$-Cbz-epicapreomycidine tert-butyl ester ((rac)-24)

General procedure B with (Z)-tert-butyl $N^{2}$-Cbz-2-amino5-azido-pent-2-enoate (Z)-21 (1.05 g, $3.04 \mathrm{mmol}), \mathrm{PPh}_{3}$ $(2.41 \mathrm{~g}, 9.18 \mathrm{mmol})$, water $(1.8 \mathrm{~mL})$ and THF $(46 \mathrm{~mL})$ for the Staudinger reduction as well as guanidinylation reagent $13(433 \mathrm{mg}, 2.96 \mathrm{mmol}), \mathrm{NEt}_{3} \quad(326 \mathrm{mg}, \quad 446 \mu \mathrm{L}$, $3.23 \mathrm{mmol})$ and DMF $(14 \mathrm{~mL})$ for the domino reaction. The reaction mixture was stirred at $80{ }^{\circ} \mathrm{C}$ for $19 \mathrm{~h}$. Purification by column chromatography $\left(\mathrm{CH}_{2} \mathrm{Cl}_{2}-\mathrm{MeOH}\right.$ gradient (8-11\%)) gave a mixture of (rac)-23 and (rac)-24 
(ratio $1.0: 1.0)$ as a brownish foam $(683 \mathrm{mg}, 62 \%$ over 2 steps from (Z)-21). ${ }^{1} \mathrm{H}$ NMR (300 $\left.\mathrm{MHz}, \mathrm{CDCl}_{3}\right): \delta=1.43$ $\left(\mathrm{s}, 2 \times 9 \mathrm{H}, t-\mathrm{Bu}_{-} \mathrm{CH}_{3}\right), 1.65-1.99(\mathrm{~m}, 2 \times 2 \mathrm{H}, \mathrm{H}-4)$, 3.13-3.26 (m, $1 \times 2 \mathrm{H}, \mathrm{H}-5), 3.26-3.42(\mathrm{~m}, 1 \times 2 \mathrm{H}$, $\mathrm{H}-5), \quad 3.70-3.80$ (m, $1 \times 1 \mathrm{H}, \mathrm{H}-3), 3.81-3.91$ (m, $1 \times 1 \mathrm{H}, \mathrm{H}-3), 4.29-4.40(\mathrm{~m}, 2 \times 1 \mathrm{H}, \mathrm{H}-2), 5.02-5.14$ $\left(\mathrm{m}, 2 \times 2 \mathrm{H}, \mathrm{Cbz}-\mathrm{CH}_{2}\right), 6.18\left(\mathrm{~d}_{\mathrm{br}}, J=8.5 \mathrm{~Hz}, 2 \times 1 \mathrm{H}\right.$, Cbz-NH), $7.03\left(\mathrm{~s}_{\mathrm{br}}, 1 \times 2 \mathrm{H}\right.$, guanidine-NH), $7.13\left(\mathrm{~s}_{\mathrm{br}}\right.$, $1 \times 2 \mathrm{H}$, guanidine-NH), 7.22-7.35 (m, $2 \times 5 \mathrm{H}$, aryl-H), $7.89\left(\mathrm{~s}_{\mathrm{br}}, 1 \times 1 \mathrm{H}\right.$, guanidine-NH), $8.02\left(\mathrm{~s}_{\mathrm{br}}, 1 \times 1 \mathrm{H}\right.$, guanidine-NH), $8.10\left(\mathrm{~s}_{\mathrm{br}}, 1 \times 1 \mathrm{H}\right.$, guanidine-NH), 8.13 $\left(\mathrm{s}_{\mathrm{br}}, \quad 1 \times 1 \mathrm{H}\right.$, guanidine-NH). ${ }^{13} \mathrm{C} \quad \mathrm{NMR} \quad(76 \mathrm{MHz}$, $\left.\mathrm{CDCl}_{3}\right): \delta=21.8(1 \times \mathrm{C}-4), 23.0(1 \times \mathrm{C}-4), 27.9(2 \times$ $t$-Bu-CH $\left.\mathrm{CH}_{3}\right), 36.6(1 \times \mathrm{C}-5), 36.7(1 \times \mathrm{C}-5), 50.2(1 \times$ C-3), $51.4(1 \times \mathrm{C}-3), 56.9(1 \times \mathrm{C}-2), 57.4(1 \times \mathrm{C}-2), 67.3$ $\left(2 \times \mathrm{Cbz}_{-} \mathrm{CH}_{2}\right), 83.8(1 \times t-\mathrm{Bu}-\mathrm{C}), 83.9(1 \times t-\mathrm{Bu}-\mathrm{C})$, $128.0 \quad(2 \times$ Cbz-aryl-C-2, $2 \times$ Cbz-aryl-C-6), 128.1 $(1 \times$ Cbz-aryl-C-4), $128.2 \quad(1 \times$ Cbz-aryl-C-4), 128.5 $(2 \times$ Cbz-aryl-C-3, $2 \times$ Cbz-aryl-C-5), $136.0(1 \times$ Cbzaryl-C-1), $136.1(1 \times$ Cbz-aryl-C-1), $154.9(1 \times$ Cbz$\mathrm{C}=\mathrm{O}), 155.0(1 \times \mathrm{Cbz}-\mathrm{C}=\mathrm{O}), 156.3(1 \times$ guanidine-C $)$, $156.6(1 \times$ guanidine-C $), 167.6(1 \times \mathrm{C}-1), 168.4(1 \times$ $\mathrm{C}-1)$. $\mathrm{MS}\left(\mathrm{ESI}^{+}\right): m / z=363.2[\mathrm{M}+\mathrm{H}]^{+}$. HRMS $\left(\mathrm{ESI}^{+}\right)$: calcd. for $\mathrm{C}_{18} \mathrm{H}_{26} \mathrm{~N}_{4} \mathrm{O}_{4}\left([\mathrm{M}+\mathrm{H}]^{+}\right)$363.2027, found 363.2029. TLC: $R_{f}=0.33\left(\mathrm{CH}_{2} \mathrm{Cl}_{2}-\mathrm{MeOH} 4: 1\right)$.

$N^{2}$-Acetyl-capreomycidine hydrochloride (( $\left.\left.r a c\right)-27\right)$ and $N^{2}$-acetyl-epicapreomycidine hydrochloride ((rac)-28)

A mixture of $N^{2}$-Cbz-capreomycidine tert-butyl ester (rac)-23 and $N^{2}$-Cbz-epicapreomycidine tert-butyl ester (rac)-24 (174 mg, $0.481 \mathrm{mmol}$ ), acetic anhydride (123 mg, $113 \mu \mathrm{L}, 1.20 \mathrm{mmol})$ and $\mathrm{Pd} / \mathrm{C}(10 \%, 13 \mathrm{mg}, 12 \mu \mathrm{mol})$ in anhydrous $\mathrm{MeOH}$ (4.8 mL) was stirred under a hydrogen atmosphere (1 bar, balloon) at rt for $22 \mathrm{~h}$. After filtration through Celite and washing the Celite with $\mathrm{MeOH}(2 \times)$, the filtrate was evaporated under reduced pressure to give a mixture of (rac)-25 and (rac)-26 containing acetic anhydride as a yellow oil (170 mg). This mixture was then dissolved in hydrochloric acid $(6 \mathrm{M}, 4.0 \mathrm{~mL}, 24 \mathrm{mmol})$, and the solution was stirred at $\mathrm{rt}$ for $3 \mathrm{~h}$. The solvent was evaporated under reduced pressure to give a mixture of (rac)-27 and (rac)-28 (ratio $1.2: 1.0$ ) as an orange foam (93 mg, $77 \%$ over 2 steps from (rac)-23 and (rac)-24). ${ }^{1} \mathrm{H}$ NMR (300 MHz, $\left.\mathrm{D}_{2} \mathrm{O}\right): \delta=1.88-2.20$ (m, $\left.2 \times 2 \mathrm{H}, \mathrm{H}-4\right)$, $2.11\left(\mathrm{~s}, 1 \times 3 \mathrm{H}, \mathrm{Ac}-\mathrm{CH}_{3}\right), 2.13\left(\mathrm{~s}, 1 \times 3 \mathrm{H}, \mathrm{Ac}-\mathrm{CH}_{3}\right)$, 3.36-3.46 (m, $2 \times 2 \mathrm{H}, \mathrm{H}-5), 4.02-4.11(\mathrm{~m}, 2 \times 1 \mathrm{H}$, $\mathrm{H}-3), 4.65-4.82(\mathrm{~m}, 2 \times 1 \mathrm{H}, \mathrm{H}-2) .{ }^{13} \mathrm{C} \mathrm{NMR}(76 \mathrm{MHz}$, $\left.\mathrm{D}_{2} \mathrm{O}\right): \quad \delta=20.7(1 \times \mathrm{C}-4), 21.7\left(1 \times \mathrm{Ac}_{-} \mathrm{CH}_{3}\right), \quad 21.8$ $\left(1 \times \mathrm{Ac}^{-\mathrm{CH}_{3}}\right), \quad 22.2(1 \times \mathrm{C}-4), \quad 36.1(1 \times \mathrm{C}-5), \quad 36.2$ $(1 \times \mathrm{C}-5), 49.3(2 \times \mathrm{C}-3), 54.7(1 \times \mathrm{C}-2), 55.2(1 \times$ C-2), $154.1(1 \times$ guanidine-C), $154.4(1 \times$ guanidine-C $)$,
$172.4(2 \times \mathrm{C}-1), \quad 174.6(2 \times \mathrm{Ac}-\mathrm{C}=\mathrm{O}) . \quad \mathrm{MS} \quad\left(\mathrm{ESI}^{+}\right)$: $m / z=237.1 \quad[\mathrm{M}+\mathrm{Na}]^{+}$. HRMS $\left(\mathrm{ESI}^{+}\right)$: calcd. for $\mathrm{C}_{8} \mathrm{H}_{14} \mathrm{~N}_{4} \mathrm{O}_{3}\left([\mathrm{M}+\mathrm{Na}]^{+}\right) 237.0958$, found 237.0958 .

X-ray structure determination of (Z)-11a

Single crystals of (Z)-11a were selected and covered with perfluorinated polyether oil on a microscope slide, which was cooled with a nitrogen gas flow using the X-TEMP2 (Kottke and Stalke 1993; Kottke et al. 1996; Stalke 1998). An appropriate crystal was selected using a polarize microscope, mounted on the tip of a MiTeGen (C-MicroMount, fixed to a goniometer head and shock-cooled by the crystal cooling device.

The data for $(Z)$-11a were collected from shock-cooled crystals at 100(2) K (Kottke and Stalke 1993; Kottke et al. 1996; Stalke 1998). The data of (Z)-11a were collected on a InCOATEC Mo Microsource (Schulz et al. 2009) with mirror optics and APEX II detector with a D8 goniometer. The diffractometer was equipped with a low-temperature device and used $\mathrm{MoK}_{\alpha}$ radiation, $\lambda=71.073 \mathrm{pm}$. The crystal of (Z)-11a used for data collection was a nonmerohedral twin with two twin domains. The two domains were separated using RLATT. Two orientation matrices were used for the integration with SAINT (Bruker AXS Inst. Inc. 2011) and a semi-empirical absorption correction with TwinABS (Sheldrick 2012) was applied. The structure was solved by direct methods (SHELXs-97) (Sheldrick 1990) using untwinned data (HKLF 4) and refined by full-matrix least-squares methods against $F^{2}$ (SHELXL-97) (Sheldrick 2008; Müller et al. 2006) using the twinned data (HKLF 5) within the SHELXLE GUI (Hübschle et al. 2011). The fractional distribution of the second domain refines to 0.4669 (9). All non-hydrogen-atoms were refined with anisotropic displacement parameters. The hydrogen atoms were refined isotropically on calculated positions using a riding model with their $U_{\text {iso }}$ values constrained to equal to 1.5 times the $U_{\text {eq }}$ of their pivot atoms for terminal $\mathrm{sp}^{3}$ carbon atoms and 1.2 times for all other carbon atoms. Only the hydrogen atoms of the nitrogen atoms ( $\mathrm{H} 1$ and $\mathrm{H} 8)$ were found from difference density map and restrained to same distance within the esd (0.02 $\AA$ ). Disordered moieties were refined using bond lengths and angles restraints and anisotropic displacement parameter restraints.

Crystallographic data (excluding structure factors) for the structures reported in this paper have been deposited with the Cambridge Crystallographic Data Centre. The CCDC number, crystal data and further details for the X-ray measurement are listed in the Supplementary Material. Copies of the data can be obtained free of charge from The Cambridge Crystallographic Data Centre via www.ccdc.cam.ac.uk/data_request/cif or from the corresponding author. 


\section{Results and discussion}

For the synthesis of potential cyclization precursors 8a-h (Table 1), a general synthetic approach was developed. In order to achieve diversity with respect to the protecting group pattern of the guanidine moiety, it was envisaged to introduce this functionality at a late stage. Guanidinylation of primary or secondary amines is a common way to prepare guanidines (for the terminology of guanidine formation, see Jones 2002). However, since $\delta$ amino-didehydro $\alpha$-amino acid esters (i.e., didehydroornithine derivatives) with an unprotected $\delta$-amino group display limited stability, the corresponding $\delta$-azido analogues were synthesized first. Reduction of the azide moiety would then provide the amines suitable for guanidinylation reactions. This approach has previously been used in the synthesis of a protected didehydro-arginine derivative (Yonezawa et al. 2000). However, to the best of our knowledge, the literature precedent for the synthesis and further transformation of a $\delta$-azido-didehydro $\alpha$-amino acid ester is limited to this one example.

Thus, rapid access to protected $\delta$-azido-didehydro $\alpha$-amino acids was an essential requirement for the described synthetic strategy. All $\delta$-azido-didehydro $\alpha$-amino acids used in this study were prepared by Wittig-Horner reactions of amino acid phosphonates 9a-c (Ducho et al. 2009; Schmidt et al. 1984) with 3-azido-propionaldehyde 10 (Davies et al. 1967) (Fig. 3). This approach proved to be significantly more efficient than the previously used lengthy transformation of protected didehydro-glutamate into the respective $\delta$-azido compound (Yonezawa et al. 2000). Wittig-Horner reactions of amino acid phosphonates with aldehydes are known to furnish the according (Z)-didehydro amino acids with excellent diastereoselectivities (Schmidt et al. 1992; for further examples, see Ducho et al. 2009; Spork and Ducho 2010; Spork et al. 2011). Accordingly, the (Z)-configured products (Z)-11ac were obtained in isolated yields of $52-77 \%$. The synthesis of cyclization precursors $\mathbf{8 b}$ and $\mathbf{8 g}, \mathbf{h}$ required the presence of a second Boc group at the $\alpha$-amino moiety. Thus, $(Z)$-11a was treated with di-tert-butyl dicarbonate $\left((\mathrm{Boc})_{2} \mathrm{O}\right)$ to afford $(Z)-\mathbf{1 1 d}$ in $92 \%$ yield. For the stereochemical assignment of $(Z)$-11a, the according $(E)$-isomer was needed as a reference compound. Following careful column chromatography, the $(E)$-configured congener $(E)$ 11a was isolated as a byproduct from the Wittig-Horner transformation of $\mathbf{9 a}$ with $\mathbf{1 0}$ in $4 \%$ yield. Application of the established ${ }^{1} \mathrm{H}$ NMR criteria for the distinction of $(Z)$ and $(E)$-didehydro amino acids (Mazurkiewicz et al. 2005) on both isomers of 11a clearly revealed the major product to display (Z)-configuration (for details see Supplementary Material). For a completely unambiguous assignment of the double-bond geometry, single crystals of $(Z)$-11a suitable

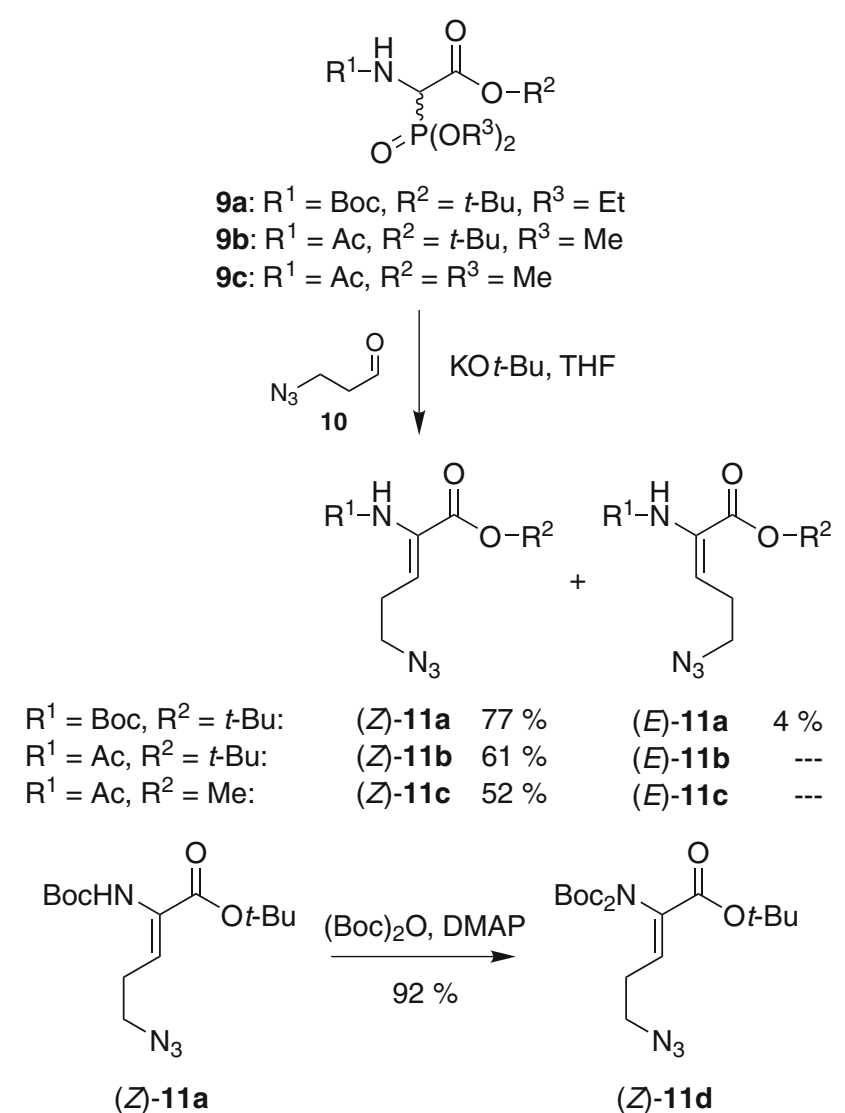

Fig. 3 Synthesis of protected $\delta$-azido-didehydro $\alpha$-amino acids $\mathbf{1 1}$

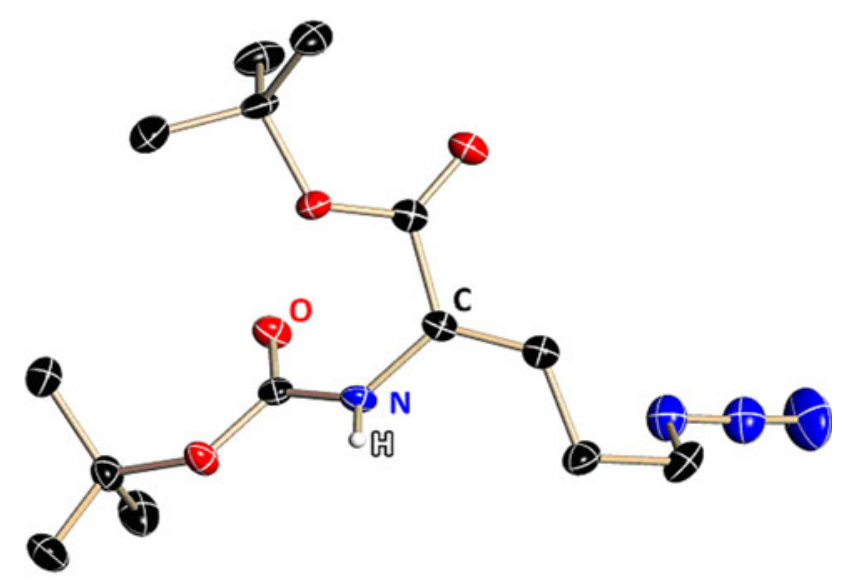

Fig. 4 Molecular structure of protected $\delta$-azido-didehydro $\alpha$-amino acid (Z)-11a. Ellipsoids are shown at $50 \%$ probability level. Carbon atoms are shown in black, nitrogen in blue, oxygen red and hydrogen white. The other hydrogen atoms and the disorder of the azide-group are omitted for clarity

for X-ray crystallography could be obtained by slow evaporation of a solution of the compound in diethyl ether. The elucidated structure confirmed the (Z)-configuration of the double bond (Fig. 4). The crystals of (Z)-11a grew as non-merohedral twins in the space group $P 2_{1} / \mathrm{c}$ with two hydrogen-bonded $(\mathrm{N}-\mathrm{H} \cdots \mathrm{O} \quad \mathrm{d}=206(2) \mathrm{pm})$ molecules 
within the asymmetric unit (see Supplementary Material). The azide groups of both molecules are disordered on two positions with an occupancy of $50 \%$.

The first attempt to achieve the desired biomimetic cyclization was to synthesize precursor 8a without protecting groups at the guanidine moiety (Fig. 5). Azido derivative (Z)-11a was therefore reduced under Staudinger conditions to give amino intermediate 12, which was not purified with respect to anticipated stability issues (see Yonezawa et al. 2000). Guanidinylation of 12 was carried out using commercially available reagent $\mathbf{1 3}$ in the presence of triethylamine to liberate the reactive agent from the employed hydrochloride. However, instead of the expected product $(Z)-\mathbf{8 a}$, a mixture of protected capreomycidine ( $\mathrm{rac}$ )-14 and epicapreomycidine ( $\mathrm{rac}$ )-15 (diastereomeric ratio (d.r.) ca. $1: 1$ ) was isolated in $54 \%$ yield over two steps from $(Z)$-11a. Thus, after formation of intermediate $(Z)-\mathbf{8 a}$, an immediate aza-Michael-addition occurred in a domino fashion (for the concept of domino reactions, see Tietze and Beifuss 1993; Tietze 1996; Tietze et al. 2006). Similar results were obtained with precursor $(Z)-\mathbf{1 1 d}$, which was reduced to ornithine derivative 16. Intermediate $\mathbf{1 6}$ was then used in the domino reaction to give a mixture of protected capreomycidine (rac)-17 and epicapreomycidine (rac)-18 (d.r. $\sim 1.8: 1.0)$ in $49 \%$ yield over two steps from (Z)-11d. Hence, the higher electrophilicity of the precursor 16 bearing two Boc groups did not result in higher isolated yields of the domino product (Fig. 5). Interestingly, when isomer $(E)$-11a was used in the sequence of Staudinger reduction and subsequent domino-guanidinylation-azaMichael-addition, no product could be isolated. It was therefore concluded that either the $(E)$-configured ornithine derivative was more instable than the $(Z)$-isomer or that the $(E)$-didehydro-arginine underwent decomposition under the reaction conditions.

The thus synthesized mixtures of protected capreomycidine (rac)-14 and epicapreomycidine ( $r a c)-\mathbf{1 5}$ as well as (rac)-17 and (rac)-18, respectively, were deprotected under acidic conditions to furnish a mixture of target compounds capreomycidine (rac)-1 and epicapreomycidine (rac)-2 as their dihydrochlorides in yields of 99 and $87 \%$, respectively (Fig. 5). A procedure for the separation of $\mathbf{1}$ and $\mathbf{2}$ by crystallization of the picrates is established (Bycroft et al. 1971b). Our novel biomimetic synthesis can therefore be considered a very convenient and rapid access to pure $\mathbf{1}$ and/or $\mathbf{2}$ in racemic form.

It was envisaged that a stereocontrolled version of the domino reaction would be difficult to achieve. First attempts to employ chiral bases such as $(-)$-quinine or $(-)-$ sparteine instead of triethylamine resulted in low conversions and side reactions. Hence, it was decided to prepare $N^{2}$-acetyl derivatives of $\mathbf{1}$ and $\mathbf{2}$ using the biomimetic route to enable acylase-mediated resolution of the product mixtures for separation of the L- from the D-isomers. Didehydro amino acids $(Z)-\mathbf{1 1 b}$ and $(Z)$-11c were therefore used in the newly established protocol of Staudinger reduction (products 19a and 19b, not purified) and subsequent biomimetic domino transformation (Fig. 6). Surprisingly, the anticipated domino process stopped at the didehydro-arginine stage after the reduction and guanidinylation steps for both precursors $(Z)-\mathbf{1 1 b}$ and (Z)-11c. Thus, compounds $8 \mathbf{c}$ and $8 \mathbf{d}$ were obtained in impure form and did not undergo cyclization towards the capreomycidine scaffold even at elevated temperatures up to $100{ }^{\circ} \mathrm{C}$. This indicates that the didehydro amino acid moiety is required to display a certain electrophilicity to enable the aza-Michael addition and that even slight changes, such as

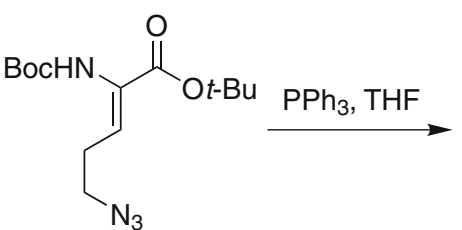

$(Z)-11 a$<smiles>CCCCOC(=O)/C(=C/CCN)NC(=O)OC</smiles>

12<smiles>CCCOC(=O)/C(=C/CCN)NC(=O)OCc1ccccc1</smiles>

$(Z)-11 d$
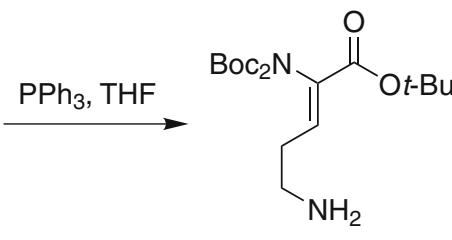

16

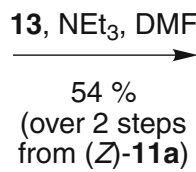<smiles>NC(=[OH+])n1cccn1</smiles>

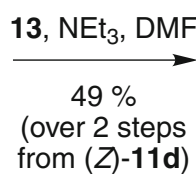

from $(Z)-11 d)$

Fig. 5 Synthesis of capreomycidine ( $r a c)-\mathbf{1}$ and epicapreomycidine ( $\mathrm{rac}$-2 $\mathbf{2}$ using a biomimetic domino reaction 
the shift from carbamate to amide protection, can lead to a complete loss of reactivity.

$\mathrm{N}$-Acetylated capreomycidin derivatives for enzymatic resolution therefore had to be prepared via a different route. An attempted direct acetylation of target structures $(r a c)-1$ and $(r a c)-2$ did not provide the desired $N^{\alpha}$-acetylated products. It was therefore envisaged to synthesize the
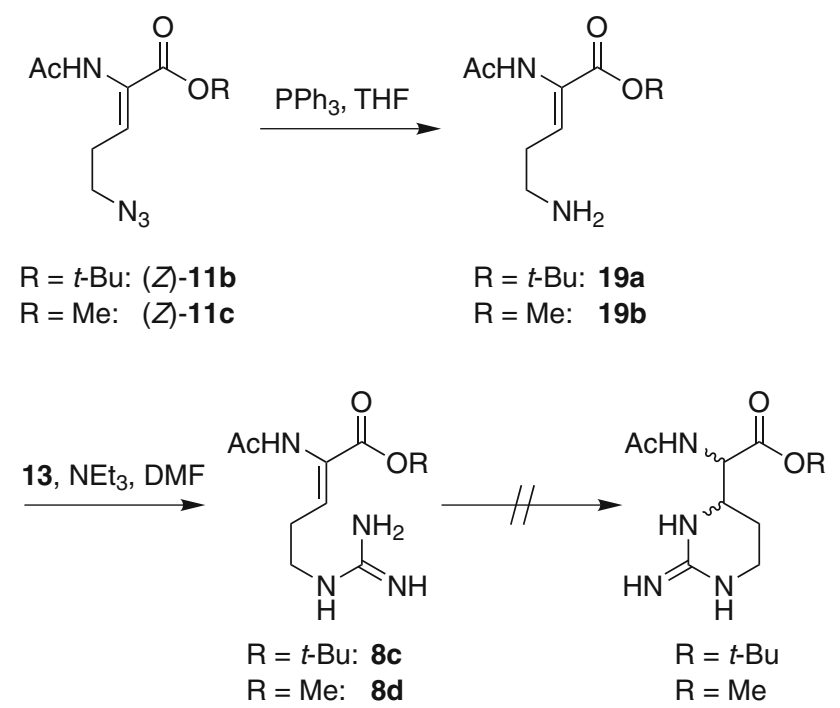

Fig. 6 Synthesis of $N^{\alpha}$-acetylated capreomycidines for enzymatic resolution: first attempt with the domino reaction stopping after the guanidinylation step according $N$-Cbz-protected congeners first and then to exchange the $N$-Cbz for an $N$-acetyl moiety after the biomimetic cyclization process, followed by acidic cleavage of the tert-butyl ester (Fig. 7). When Cbz-protected phosphonate 20 (Schmidt et al. 1982, 1984; Schmidt and Wild 1985; Hamzavi et al. 2003) was employed in the WittigHorner reaction with aldehyde 10, Cbz-protected $\delta$-azidodidehydro $\alpha$-amino acid ester $(Z)$-21 was obtained in $81 \%$ yield (calculated as traces of aldehyde $\mathbf{1 0}$ could not completely be removed). Stereoisomer $(E)$-21 could also be isolated in $3 \%$ yield, and again, application of the established ${ }^{1} \mathrm{H}$ NMR criteria for the distinction of $(Z)$ - and (E)-didehydro amino acids (Mazurkiewicz et al. 2005) confirmed the stereochemical assignment. Subsequent Staudinger reduction of (Z)-21 furnished ornithine derivative 22, which was directly treated with guanidinylation reagent $\mathbf{1 3}$ to initiate the domino reaction. Thus, formation of the respective arginine derivative and domino-type ring closure provided a mixture of protected capreomycidine (rac)-23 and epicapreomycidine (rac)-24 (d.r. $\sim 1: 1)$ in $62 \%$ yield over two steps. The mixture of (rac)-23 and (rac)-24 was then subjected to a sequence of hydrogenolysis with in situ-acetylation (products (rac)-25 and (rac)-26) and acidic cleavage of the tert-butyl ester to afford a mixture of $N^{\alpha}$-acetylated products $(\mathrm{rac})-27$ and (rac)-28 in $77 \%$ yield over two steps from the mixture of (rac)-23 and (rac)-24 (Fig. 7). However, attempts to

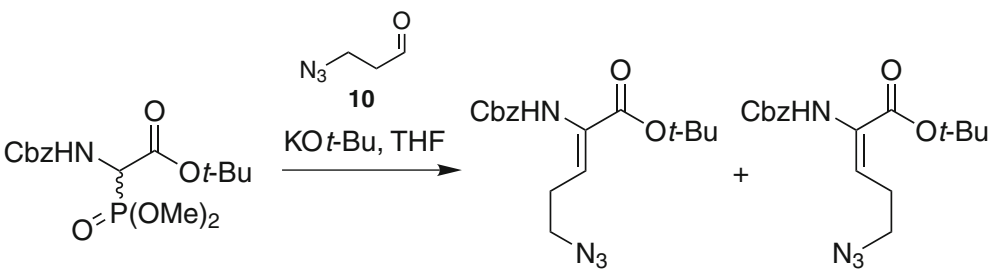

20

(Z)-21 $81 \%$

(E)-21 $3 \%$<smiles>CCOC(=O)/C(=C\CCN)NC(=O)OCc1ccccc1</smiles>

$(Z)-21$<smiles>CCOC(=O)C(=CCCN)NC(=O)OCc1ccccc1</smiles>

22

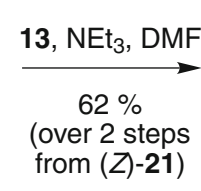

(over 2 steps
from (Z)-21)

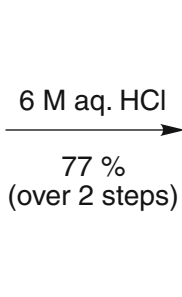<smiles>CCCCOC(=O)[C@H](NC(=O)OCc1ccccc1)[C@@H]1CCNC(=N)N1</smiles>

(rac)-23<smiles>CCC(C)OC(=O)[C@H](NC(=O)OCc1ccccc1)[C@@H]1CCNC(=N)N1</smiles>

(rac)-24

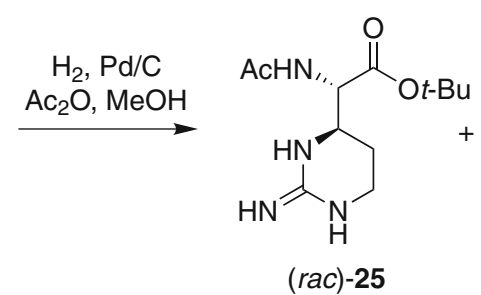<smiles>C=CN[C@H](C(=O)OCCCC)[C@@H]1CCNC(=N)N1</smiles><smiles>N=C1NCC[C@H]([C@H](N=CCl)C(=O)O)[NH2+]1</smiles>

Fig. 7 Synthesis of $N^{\alpha}$-acetylated capreomycidines for enzymatic resolution: successful route via $N^{\alpha}$-Cbz-protected derivatives 
subject this mixture of $N^{\alpha}$-acetylated capreomycidines to acylase-mediated deacetylation to separate the L- from the D-isomers failed as no conversion could be detected. Apparently, $N^{\alpha}$-acetylated capreomycidines are no substrates for standard acylases. This might result either from steric hindrance due to the adjacent six-membered ring or from the positively charged guanidine group being fixed in a position close to the reaction site.

The synthesis of guanidine-protected cyclization precursors 8e-h (see Table 1) was then attempted. It was anticipated that these compounds should not spontaneously undergo the aza-Michael addition as a result from the reduced nucleophilicity of the guanidine group. In contrast, one should be able to isolate fully protected didehydroarginine derivatives $\mathbf{8 e}-\mathbf{h}$ and to subject them to azaMichael cyclization in a separate step. This second reaction would then require activation either with a Lewis acid (in order to enhance the electrophilicity of the didehydro amino acid moiety) or a base (to deprotonate the urethaneprotected guanidine and make it nucleophilic). Thus, the domino reaction would be dissected into two separate transformations, and a chiral activating agent for the cyclization step might potentially provide stereoinduction and enable a stereoselective biomimetic synthesis of capreomycidines. In principle, the acidic properties of urethane-protected guanidines are established (Feichtinger et al. 1998). However, it was unclear if the guanidine moiety had to be fully protected in order to allow for sufficient deprotonation of the urethane moiety, and therefore, the additional $N$-benzyl group was introduced into potential cyclization precursors $8 \mathbf{f}$ and $\mathbf{8 h}$.

For an efficient synthesis of $\mathbf{8 e}-\mathbf{h}, \delta$-azido-didehydro $\alpha$-amino acids (Z)-11a and (Z)-11d were used and again reduced to moderately stable ornithine derivatives $\mathbf{1 2}$ and 16 (vide supra, Fig. 8). For the introduction of the benzyl group, 12 and 16 were subjected to reductive amination reactions with benzaldehyde to furnish $N^{\delta}$-benzylated ornithines 29 and 30, which could not be obtained in pure form. All didehydro-ornithines 12, 16, 29 and 30 were transformed into the respective arginine derivatives by guanidinylation with reagent 31 (Tian et al. 1992) in the presence of silver(I) triflate. Thus, target structures 8eh were obtained in yields of $42-71 \%$ (over two or three steps from the respective $\delta$-azido-didehydro $\alpha$-amino acid).

Guanidine-protected cyclization precursors $\mathbf{8 e}-\mathbf{h}$ were then subjected to numerous attempts to activate them for the aza-Michael addition. Initially, 8e was treated with a series of bases $\left(\mathrm{K}_{2} \mathrm{CO}_{3}\right.$ in $\mathrm{MeCN}, \mathrm{NaH}$, KHMDS or DBU in THF) for urethane deprotonation or with iron(III) chloride as a Lewis acid in dichloromethane for activation of the Michael acceptor system (reactions not displayed). No conversion towards the cyclic capreomycidine system was detected. As the bis-Boc-protected congener $\mathbf{8 g}$ was

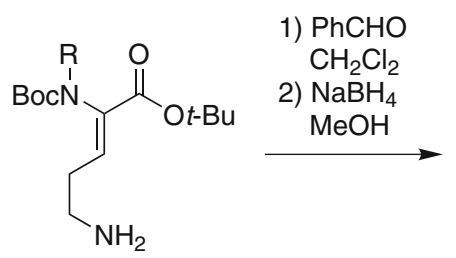

$$
\left.\begin{array}{lr}
R=H: & 12 \\
R & =\text { Boc: } 16
\end{array}\right\} \begin{aligned}
& \text { obtained by } \\
& \text { reduction of } \\
& (Z)-11 a, d
\end{aligned}
$$

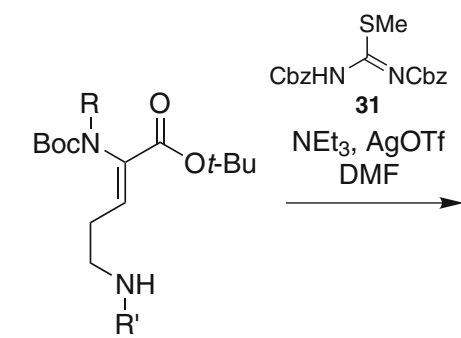

$\mathrm{R}=\mathrm{R}^{\prime}=\mathrm{H}: \quad 12$

$\mathrm{R}=\mathrm{H}, \mathrm{R}^{\prime}=\mathrm{Bn}: \quad 29$

$\mathrm{R}=\mathrm{Boc}, \mathrm{R}^{\prime}=\mathrm{H}: \quad 16$

$\mathrm{R}=\mathrm{Boc}, \mathrm{R}^{\prime}=\mathrm{Bn}: 30$

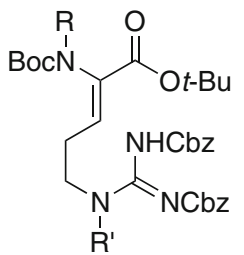

$\mathrm{R}=\mathrm{H}: \quad 29$

$\mathrm{R}=$ Boc: $\mathbf{3 0}$

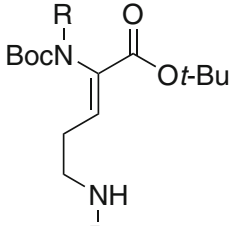

$\mathrm{R}=\mathrm{R}^{\prime}=\mathrm{H}: \quad \mathbf{8 e} \quad 71 \%$ *

$\mathrm{R}=\mathrm{H}, \mathrm{R}^{\prime}=\mathrm{Bn}: \quad$ 8f $\quad 55 \%$ **

$\mathrm{R}=\mathrm{Boc}, \mathrm{R}^{\prime}=\mathrm{H}: \mathbf{8 g} 50 \%$ *

$\mathrm{R}=\mathrm{Boc}, \mathrm{R}^{\prime}=\mathrm{Bn}: \mathbf{8 h} 42 \%$ **

*over 2 steps from (Z)-11a,d

**over 3 steps from (Z)-11a,d

Fig. 8 Synthesis of potential cyclization precursors $8 \mathbf{e}-\mathbf{h}$ with protected guanidine moieties

anticipated to be more reactive, its possible cyclization in the presence of DBU in THF was investigated next, but no conversion was observed even at elevated temperatures. It was therefore decided to skip cyclization studies on $\mathbf{8 f}$ and to conduct more detailed investigations regarding the azaMichael cyclization of what should be the most reactive precursor both in terms of guanidine-urethane acidity and Michael acceptor activity, i.e. didehydro-arginine $\mathbf{8 h}$. Hence, compound $\mathbf{8 h}$ was treated with a series of bases $\left(\mathrm{K}_{2} \mathrm{CO}_{3}\right.$ in DMSO, basic $\mathrm{Al}_{2} \mathrm{O}_{3}$ in $\mathrm{MeCN}, \mathrm{KO} t$-Bu or $\mathrm{NaH}$ in THF) as well as a series of Lewis acids $\left(\mathrm{FeCl}_{3}, \mathrm{SnCl}_{4}\right.$, $\mathrm{Me}_{2} \mathrm{AlCl}, \mathrm{EtAlCl}_{2}$ or $\mathrm{Yb}(\mathrm{OTf})_{3}$ in dichloromethane, reactions not displayed). Furthermore, Takemoto's bifunctional chiral organocatalyst (Inokuma et al. 2006) was added to a solution of $\mathbf{8 h}$ in toluene to study if the combination of thiourea activation and basic moiety might be able to trigger the cyclization reaction. However, no conversion towards the cyclic capreomycidine system was detected in any of the listed cases. It was therefore concluded that the reactivity of the didehydro amino acid moiety was just not sufficient for aza-Michael additions if the nucleophilicity of the guanidine group is too low resulting from its derivatization. This again demonstrated that the biomimetic cyclization reaction could only work within a certain frame of reactivity of both moieties involved (vide supra). Thus, a dissection of the domino reaction into two separate transformations was impossible, preventing further 
attempts to conduct the biomimetic cyclization in a stereocontrolled fashion.

\section{Conclusion}

In summary, we have developed an unprecedented biomimetic pathway for the concise synthesis of the non-proteinogenic amino acids capreomycidine $\mathbf{1}$ and epicapreomycidine $\mathbf{2}$ in racemic form. The two key steps of this efficient synthetic route were (1) the preparation of protected $\delta$-azido-didehydro $\alpha$-amino acid precursors using Wittig-Horner reactions and (2) a sequence of Staudinger reduction and a novel domino reaction for the construction of the capreomycidine scaffold. This domino-guanidinylation-aza-Michael-addition sequence mimics the biosynthetic ring closure of didehydro-arginine towards capreomycidine. Attempts to subject the furnished racemic mixtures of $\mathrm{L}$ - and D-amino acids to acylase-mediated enzymatic resolution were unsuccessful as $N^{\alpha}$-acetylated capreomycidines were apparently no substrates for acylases. Investigations on didehydro-arginine cyclization precursors with different protecting group patterns revealed that the domino reaction would only work within a certain frame of reactivity, i.e. both sufficient electrophilicity of the Michael acceptor and nucleophilicity of the guanidine moiety were essential. Fully protected didehydro-arginine derivatives $\mathbf{8 e}-\mathbf{h}$ therefore did not undergo the aza-Michael addition anymore, even in the presence of highly reactive activating agents. However, both compounds $8 \mathbf{e}-\mathbf{h}$ and their respective azide precursors might be useful synthetic intermediates for the preparation of isotope-labelled ornithine or arginine derivatives (also see Baldwin et al. 1993a, b). Overall, the obtained results demonstrate that a biomimetic 1,4-addition of a guanidine moiety to a didehydro amino acid unit is synthetically feasible, but that a fine-tuning of the reactivity is essential.

Acknowledgments This work was supported by the Deutsche Forschungsgemeinschaft (DFG, SFB 803 "Functionality controlled by organization in and between membranes") and the Fonds der Chemischen Industrie (FCI, Sachkostenzuschuss). MG and DS thank the DFG Priority Programme 1178 and the Danish National Research Foundation (DNRF) funded Center for Materials Crystallography (CMC).

Conflict of interest The authors declare that they have no conflict of interest.

\section{References}

Ando T, Matsuura K, Izumi R, Noda T, Take T, Nagata A, Abe J (1971) Studies on tuberactinomycin. II. Isolation and properties of tuberactinomycin-N, a new tuberactinomycin group antibiotic. J Antibiot 24:680-686
Baldwin JE, Merritt KD, Schofield CJ (1993a) Synthesis of L-ornithines stereospecifically deuterated at C-3. Tetrahedron Lett 34:3919-3920

Baldwin JE, Merritt KD, Schofield CJ, Elson SW, Baggaley KH (1993b) Studies on the stereospecificity of the Clavaminic acid synthase catalysed hydroxylation reaction. J Chem Soc Chem Commun 1993:1301-1302

Bartz QR, Ehrlich J, Mold JD, Penner MA, Smith RM (1951) Viomycin, a new tuberculostatic antibiotic. Amer Rev Tuberculosis 63:4-6

Bruker AXS Inst. Inc. (2011) SAINT v7.68A in Bruker APEX v2011.9, Bruker AXS Inst. Inc., Madison, USA

Bycroft BW, Croft LR, Johnson AW, Webb T (1969) Structure, stereochemistry, and reactions of the guanidine moiety of viomycin. J Antibiot 22:133-134

Bycroft BW, Cameron D, Croft LR, Hassanali-Walji A, Johnson AW, Webb T (1971a) Total structure of capreomycin IB, a tuberculostatic peptide antibiotic. Nature 231:301-302

Bycroft BW, Cameron D, Johnson AW (1971b) Synthesis of capreomycidine and epicapreomycidine, the epimers of $\alpha-(2$ iminohexahydro-4-pyrimidyl)glycine. J Chem Soc C 1971: 3040-3047

Cheng L, Chen W, Zhai L, Xu D, Huang T, Lin S, Zhou X, Deng Z (2011) Identification of the gene cluster involved in muraymycin biosynthesis from Streptomyces sp. NRRL 30471. Mol BioSyst 7:920-927

Cooper MA, Shlaes D (2011) Fix the antibiotics pipeline. Nature $472: 32$

Davies AJ, Donald ASR, Marks RE (1967) The acid-catalysed decomposition of some $\beta$-azido-carbonyl compounds. J Chem Soc C 1967:2109-2112

DeMong DE, Williams RM (2001) The asymmetric synthesis of $(2 S, 3 R)$-capreomycidine. Tetrahedron Lett 42:3529-3532

DeMong DE, Williams RM (2003) Asymmetric synthesis of $(2 S, 3 R)$ capreomycidine and the total synthesis of capreomycin IB. J Am Chem Soc 125:8561-8565

Ducho C, Hamed RB, Batchelar ET, Sorensen JL, Odell B, Schofield CJ (2009) Synthesis of regio- and stereoselectively deuteriumlabelled derivatives of L-glutamate semialdehyde for studies on carbapenem biosynthesis. Org Biomol Chem 7:2770-2779

Dyer JR, Kellogg CK, Nassar RF, Streetman WE (1965) Viomycin II. Structure of viomycin. Tetrahedron Lett 6:585-592

Feichtinger K, Sings HL, Baker TJ, Matthews K, Goodman M (1998) Triurethane-protected guanidines and triflyldiurethane-protected guanidines: new reagents for guanidinylation reactions. J Org Chem 63:8432-8439

Finlay AC, Hobby GL, Hochstein F, Lees TM, Lenert TF, Means JA, P'An SY, Regna PP, Routien JB, Sobin BA, Tate KB, Kane JH (1951) Viomycin, a new antibiotic active against mycobacteria. Amer Rev Tuberculosis 63:1-3

Hamzavi R, Dolle F, Tavitian B, Dahl O, Nielsen PE (2003) Modulation of the pharmacokinetic properties of PNA: preparation of galactosyl, mannosyl, fucosyl, $n$-acetylgalactosaminyl, and $n$-acetylglucosaminyl derivatives of aminoethylglycine peptide nucleic acid monomers and their incorporation into PNA oligomers. Bioconjugate Chem 14:941-954

He H, Williamson RT, Shen B, Graziani EI, Yang HY, Sakya SM, Petersen PJ, Carter GT (2002) Mannopeptimycins, novel antibacterial glycopeptides from Streptomyces hygroscopicus. J Am Chem Soc 124:9729-9736

Herr EB, Haney ME, Pittenger GE, Higgens CE (1960) Isolation and characterization of a new peptide antibiotic. Proc Ind Acad Sci 69:134

Hübschle CB, Sheldrick GM, Dittrich B (2011) ShelXle: a Qt graphical user interface for SHELXL. J Appl Cryst 44:12811284 
Inokuma T, Hoashi Y, Takemoto Y (2006) Thiourea-catalyzed asymmetric michael addition of activated methylene compounds to $\alpha, \beta$-unsaturated imides: dual activation of imide by intra- and intermolecular hydrogen bonding. J Am Chem Soc 128:94139419

Izumi R, Noda T, Ando T, Take T, Nagata A (1972) Studies on tuberactinomycin. 3. Isolation and characterization of two minor components, tuberactinomycin $\mathrm{B}$ and tuberactinomycin $\mathrm{O}$. J Antibiot 25:201-207

Jackson MD, Gould SJ, Zabriskie TM (2002) Studies on the formation and incorporation of streptolidine in the biosynthesis of the peptidyl nucleoside antibiotic streptothricin F. J Org Chem 67:2934-3941

Jones JH (2002) The terminology of guanidine formation. J Pept Sci $8: 285-287$

Ju J, Ozanick SG, Shen B, Thomas MG (2004) Conversion of (2S)arginine to $(2 S, 3 R)$-capreomycidine by VioC and VioD from the viomycin biosynthetic pathway of Streptomyces $s p$. strain ATCC11861. ChemBioChem 5:1281-1285

Koehn FE (2008) New strategies and methods in the discovery of natural product anti-infective agents: the mannopeptimycins. J Med Chem 51:2613-2617

Kottke T, Stalke D (1993) Crystal handling at low temperature. J Appl Crystallogr 26:615-619

Kottke T, Lagow RJ, Stalke D (1996) Low cost conversion of a coaxial nozzle arrangement into a stationary low temperature attachment. J Appl Crystallogr 29:465-468

Lemke A, Büschleb M, Ducho C (2010) Concise synthesis of both diastereomers of 3-hydroxy-L-arginine. Tetrahedron 66:208-214

Mahady GB, Huang Y, Doyle BJ, Locklear T (2008) Natural products as antibacterial agents. Stud Nat Prod Chem 35:423-444

Mazurkiewicz R, Kuźnik A, Grymel M, Kuźnik N (2005) ${ }^{1} \mathrm{H}$ NMR spectroscopic criteria for the configuration of $N$-acyl- $\alpha, \beta$-dehydro- $\alpha$-amino acid esters. Magn Reson Chem 43:36-40

McDonald LA, Barbieri LR, Carter GT, Lenoy E, Lotvin J, Petersen PJ, Siegel MM, Singh G, Williamson RT (2002) Structures of the muraymycins, novel peptidoglycan biosynthesis inhibitors. J Am Chem Soc 124:10260-10261

Müller P, Herbst-Irmer R, Spek AL, Schneider TR, Sawaya MR (2006) In: Müller P (ed) Crystal structure refinement: a crystallographer's guide to SHELXL, IUCr texts on crystallography, vol 8. Oxford University Press, Oxford

Nagata A, Ando T, Izumi R, Sakakibara H, Take T, Hayano K, Abe J (1968) Studies on tuberactinomycin (tuberactin), a new antibiotic. I. Taxonomy of producing strain, isolation and characterization. J Antibiot 21:681-687

Noda T, Take T, Nagata A, Wakamiya T, Shiba T (1972) Chemical studies on tuberactinomycin. III. Chemical structure of viomycin (tuberactinomycin B). J Antibiot 25:427-428

Nomoto S, Teshima T, Wakamiya T, Shiba T (1977) The revised structure of capreomycin. J Antibiot 30:955-959

Nomoto S, Teshima T, Wakamiya T, Shiba T (1978) Total synthesis of capreomycin. Tetrahedron 34:921-927

Okura A, Morishima H, Takita T, Aoyagi T, Takeuchi T, Umezawa H (1975) The structure of elastatinal, an elastase inhibitor of microbial origin. J Antibiot 28:337-339

Razzak M, De Brabander JK (2011) Lessons and revelations from biomimetic syntheses. Nat Chem Biol 7:865-875

Schmidt U, Wild J (1985) Synthesen von Peptidalkaloiden, 11. Über Aminosäuren und Peptide, 51-Dehydroaminosäuren, 19. Totalsynthese von Hexaacetylcelenamid A. Liebigs Ann Chem 1985:1882-1894

Schmidt U, Lieberknecht A, Schanbacher U, Beuttler T, Wild J (1982) Facile preparation of $N$-acyl-2-(diethoxyphosphoryl) glycine esters and their use in the synthesis of dehydroamino acid esters. Angew Chem Int Ed 21:776-777
Schmidt U, Lieberknecht A, Wild J (1984) Amino acids and peptides; XLIII. dehydroamino acids; XVIII. Synthesis of dehydroamino acids and amino acids from $N$-acyl-2-(dialkyloxyphosphinyl)glycin esters; II. Synthesis 1984:53-60

Schmidt U, Griesser H, Leitenberger V, Lieberknecht A, Mangold R, Meyer R, Riedl B (1992) Diastereoselective formation of (Z)-didehydroamino acid esters. Synthesis 1992:487-450

Schulz T, Meindl K, Leusser D, Stern D, Graf J, Michaelsen C, Ruf M, Sheldrick GM, Stalke D (2009) A comparison of a microfocus X-ray source and a conventional sealed tube for crystal structure determination. J Appl Crystallogr 42:885-891

Schwörer CJ, Oberthür M (2009) Synthesis of highly functionalized amino acids: an expedient access to L- and D- $\beta$-hydroxyenduracididine derivatives. Eur J Org Chem 2009:6129-6139

Seiple IB, Su S, Young IS, Nakamura A, Yamaguchi J, Jorgensen L, Rodriguez RA, O'Malley DP, Gaich T, Kock M, Baran PS (2011) Enantioselective total syntheses of (-)-palau'amine, (-)-axinellamines, and (-)-massadines. J Am Chem Soc 133:14710-14726

Sheldrick GM (1990) Phase annealing in SHELX-90: direct methods for larger structures. Acta Crystallogr Sect A 46:467-473

Sheldrick GM (2008) A short history of SHELX. Acta Crystallogr Sect A 64:112-122

Sheldrick GM (2012) TWINABS 2012/1, Georg-August-University Göttingen, Göttingen, Germany

Shiba T, Nomoto S, Teshima T, Wakamiya T (1976) Revised structure and total synthesis of capreomycin. Tetrahedron Lett 17:3907-3910

Shiba T, Ukita T, Mizuno K, Teshima T, Wakamiya T (1977) Total synthesis of L-capreomycidine. Tetrahedron Lett 18:2681-2684

Singh MP, Petersen PJ, Weiss WJ, Janso JE, Luckman SW, Lenoy EB, Bradford PA, Testa RT, Greenstein M (2003) Mannopeptimycins, new cyclic glycopeptide antibiotics produced by Streptomyces hygroscopicus LL-AC98: antibacterial and mechanistic activities. Antimicrob Agents Chemother 47:62-69

Spork AP, Ducho C (2010) Novel 5'-deoxy nucleosyl amino acid scaffolds for the synthesis of muraymycin analogues. Org Biomol Chem 8:2323-2326

Spork AP, Wiegmann D, Granitzka M, Stalke D, Ducho C (2011) Stereoselective synthesis of uridine-derived nucleosyl amino acids. J Org Chem 76:10083-10098

Stalke D (1998) Cryo crystal structure determination and application to intermediates. Chem Soc Rev 27:171-178

Tanino T, Hirano S, Ichikawa S, Matsuda A (2008) Synthetic study of muraymycins using Ugi-four component reaction. Nucleic Acids Symp Series 52:557-558

Tanino T, Ichikawa S, Shiro M, Matsuda A (2010) Total synthesis of (-)-muraymycin D2 and its epimer. J Org Chem 75:1366-1377

Tanino T, Ichikawa S, Matsuda A (2011) Synthesis of L-epicapreomycidine derivatives via $\mathrm{C}-\mathrm{H}$ amination. Org Lett 13:4028-4031

Tatsuta K, Mikami N, Fujimoto K, Umezawa S, Umezawa H, Aoyagi $\mathrm{T}$ (1973) The structure of chymostatin, a chymotrypsin inhibitor. J Antibiot 26:625-646

Taubes G (2008) The bacteria fight back. Science 321:356-361

Teshima T, Konishi K, Shiba T (1980) Synthesis of L-epicapreomycidine. Bull Chem Soc Jpn 53:508-511

Tian Z, Edwards P, Roeske RW (1992) Synthesis of optically pure $\mathrm{C}^{\alpha}$-methyl-arginine. Int J Pept Protein Res 40:119-126

Tietze LF (1996) Domino reactions in organic synthesis. Chem Rev 96:115-136

Tietze LF, Beifuss U (1993) Sequential transformations in organic chemistry: a synthetic strategy with a future. Angew Chem Int Ed 32:131-163

Tietze LF, Brasche G, Gericke K (2006) Domino reactions in organic synthesis. Wiley-VCH, Weinheim 
Umezawa H, Aoyagi T, Morishima H, Kunimoto S, Matsuzaki M, Hamada M, Takeuchi T (1970) Chymostatin, a new chymotrypsin inhibitor produced by actinomycetes. J Antibiot 23:425-427

Umezawa H, Aoyagi T, Okura A, Morishima H, Takeuchi T, Okami Y (1973) Elastatinal, a new elastase inhibitor produced by actinomycetes. J Antibiot 26:787-789

Wakamiya T, Shiba T, Kaneko T, Sakakibara H, Take T, Abe J (1970) Chemical studies on tuberactinomycin. I. The structure of tuberactidine, guanidino amino acid component. Tetrahedron Lett 11:3497-3500

Wakamiya T, Mizuno K, Ukita T, Teshima T, Shiba T (1978) Chemical studies on tuberactinomycin. XIV. Novel synthesis of DL-capreomycidine. Bull Chem Soc Jpn 51:850-854

Walsh C (2003) Where will new antibiotics come from? Nat Rev Microbiol 1:65-70

Yamashita A, Norton EB, Mansour TS (2004) Improved procedures for preparation of racemic capreomycidine. Synth Commun 5:795-803
Yin X, Zabriskie TM (2004) VioC is a non-heme iron, $\alpha$-ketoglutarate-dependent oxygenase that catalyzes the formation of $3 S$ hydroxy-L-arginine during viomycin biosynthesis. ChemBioChem 5:1274-1277

Yin X, McPhail KL, Kim K, Zabriskie TM (2004) Formation of the nonproteinogenic amino acid $2 S, 3 R$-capreomycidine by VioD from the viomycin biosynthesis pathway. ChemBioChem $5: 1278-1281$

Yonezawa Y, Hirosaki T, Hayashi T, Shin CG (2000) Convenient synthesis and conversion of a $(Z)-\alpha, \beta$-didehydroornithine derivative to $\alpha, \beta$-didehydrokyotorphin. Synthesis 2000:144-148

Yoshioka H, Aoki T, Goko H, Nakatsu K, Noda T, Sakakibara H, Take T, Nagata A, Abe J, Wakamiya T, Shiba T, Kaneko T (1971) Chemical studies on tuberactinomycin. II Structure of tuberactinomycin O. Tetrahedron Lett 12:2043-2046 